INSTITUT NATIONAL DE LA STATISTIQUE ET DES ETUDES ECONOMIQUES

Série des Documents de Travail du CREST

(Centre de Recherche en Economie et Statistique)

\author{
$n^{\circ}$ 2006-29 \\ Multi-Lag Term Structure \\ Models with Stochastic \\ Risk Premia
}

A. MONFORT
F. PEGORARO

Les documents de travail ne reflètent pas la position de l'INSEE et n'engagent que leurs auteurs.

Working papers do not reflect the position of INSEE but only the views of the authors.

\footnotetext{
1 CNAM and IRIA. bertholo@cnam.fr and henri.bertholon@inrialpes.fr

2 CNAM and CREST, Laboratoire de Finance-Assurance. monfort@cnam.fr and monfort@ensae.fr
} 


\title{
Multi-Lag Term Structure Models with Stochastic Risk Premia
}

Alain MONFORT ${ }^{(1)}$ Fulvio PEGORARO ${ }^{(2)}$

This version : November, 2006

\author{
Abstract \\ Multi-Lag Term Structure Models with Stochastic Risk Premia
}

In this paper we propose a family of discrete-time term structure models where we specify a Gaussian autoregressive of order $p>1$ historical and risk-neutral dynamics for the factor $\left(x_{t}\right)$, considered as a latent or observable variable: in the second case the factor is a vector of several yields. We present the Gaussian $\operatorname{AR}(p)$ Factor-Based Term Structure Model in which the stochastic discount factor (SDF) for the period $(t, t+1)$ is specified as an exponential-affine function of $x_{t+1}$, the factor risk-correction coefficient is stochastic, and the associated yield-to-maturity formula at time $t$ is an affine function of $X_{t}=\left(x_{t}, \ldots, x_{t-p+1}\right)^{\prime}$. We propose the Moving Average (or Heath, Jarrow and Morton) characterization of the yield and short-term forward rate processes, under the risk-neutral and the $S$-forward probability : this representation gives the possibility to exactly replicate the currently-observed yield curve. We also study the problem of matching the theoretical and the currently-observed market term structure by means of the Extended $\operatorname{AR}(p)$ approach. We present the Gaussian $\operatorname{VAR}(p)$ Factor-Based Term Structure Model, generalizing the previously mentioned results to the multivariate framework.

Keywords : Discrete-time Affine Term Structure Models, Stochastic Discount Factor, Gaussian VAR $(p)$ processes, Stochastic risk premia, Moving Average or HJM representations, Exact Fitting of the currently-observed yield curve.

\section{Résumé}

\section{Modèles de Structure par Terme à Plusieurs Retards et Primes de Risques Stochastiques}

Let but de ce papier est de proposer une famille de modèles en temps discret, pour la construction de la courbe de taux d'intérêt, dans laquelle les dynamiques historique et risque-neutre du facteur $\left(x_{t}\right)$ sont représentées par un processus autoregressif Gaussien d'ordre $p>1$. Le facteur peut une variable latente ou une variable observable : dans ce deuxième cas le facteur sera un vecteur de taux de différentes maturités. On présente le Modèle de Structure par Terme $\operatorname{AR}(p)$ Gaussien, dans lequel le facteur d'escompte stochastique (SDF) pour la période $(t, t+1)$ est une fonction exponentielle-affine du facteur $x_{t+1}$, le coefficient d'ajustement pour le risque du facteur est stochastique, et la formule des taux à la date $t$ est une fonction affine du vecteur $X_{t}=\left(x_{t}, \ldots, x_{t-p+1}\right)^{\prime}$. On propose la caractérisation Moyenne Mobile (ou Heath, Jarrow and Morton) des processus des taux et des taux forwards à court terme : cette représentation donne la possibilité de répliquer exactement la courbe des taux observée dans le marché. On étudie aussi le problème de la réplication de la courbe de taux du marché à l'aide de l'approche $\operatorname{Extended~} \operatorname{AR}(p)$. On présente le Modèle de Structure par Terme $\operatorname{VAR}(p)$ Gaussien, en généralisant au contexte multivarié les résultats précédemment mentionnés.

Mots Clés : Modèles Affines en temps discret pour la courbe de taux d'intérêt, Facteur d'Escompte Stochastique, processus VAR(p) Gaussien, Prime de Risque Stochastique, représentations Moyenne Mobile ou HJM, Réplication exacte de la courbe de taux du marché.

JEL number : C1, C5, G1

\footnotetext{
${ }^{1}$ CNAM [E-mail: monfort@cnam.fr] and CREST, Laboratoire de Finance-Assurance [E-mail: monfort@ensae.fr]. ${ }^{2}$ Banque de France, Economics and Finance Research Center [DGEI-DIR-RECFIN; E-mail: Fulvio.PEGORARO@banque-france.fr] and CREST, Laboratoire de Finance-Assurance [E-mail: pegoraro@ensae.fr].
} 


\section{Introduction}

One of the most important Affine Term Structure Models was the one proposed by Vasicek (1977) in a famous paper where the factor driving the specification of the entire interest rate curve was the instantaneous spot interest rate $r=\left(r_{t}, t \geq 0\right)$. The model was defined in a continuoustime framework and the dynamics of $\left(r_{t}\right)$ was described, under the historical probability, by an Ornstein-Uhlenbeck process with constant coefficients [the continuous-time equivalent of a discretetime Gaussian AR(1) process]. The limits of this model are well-known: for each time $t$ the rate $r_{t}$ can be negative with positive probability and the term structure can show a limited number of shapes [monotone increasing, constant, monotone decreasing and humpshaped].

The first drawback is compensated by the analytical tractability, induced by the Gaussian (historical and risk-neutral) dynamics of the factor, which is hardly achieved when other conditional distributions are considered for the process $\left(r_{t}\right)$ : indeed, the success of this model, along with the Cox, Ingersoll and Ross (1985) model, comes from the possibility to provide explicit or quasi explicit pricing formula for zero-coupon bonds and interest rate derivatives.

With regard to the second drawback, the continuous-time approach can generalize the yield-tomaturity formula only by the introduction of other (observable or latent) factors [see, among the others, Pennacchi (1991), Duffie and Kan (1996), Chen (1996), Dai and Singleton (2000, 2003), Duffee (2002), Brandt and Chapman (2002), Duarte (2004), Berardi (2005), Berardi and Torous (2005), Cheridito, Filipovic and Kimmel (2006)]. On the contrary, the discrete-time approach we follow in this paper is characterized by an additional degree of freedom: the possibility to specify an autoregressive of order $p>1$ dynamics for the factor driving the term structure shapes.

We propose discrete-time term structure models where the historical dynamics of the factor $\left(x_{t}\right)$ is given, in the univariate case, by a Gaussian $\operatorname{AR}(p)$ process, and, in the multivariate case, by a Gaussian $n$-dimensional $\operatorname{VAR}(p)$ process. The factor $\left(x_{t}\right)$ is considered as a latent or an observable variable : in the second case $\left(x_{t}\right)$ is a vector of several yields. We consider an exponential-affine SDF, with a stochastic risk premium defined, at time $t$, as an affine function of $X_{t}=\left(x_{t}, \ldots, x_{t-p+1}\right)^{\prime}$ and, consequently, the yield-to-maturity formula at time $t$ is an affine function of the $p$ most recent lagged values of $x_{t+1}$.

Compared with the continuous-time affine case, our approach proposes a more general specification of the conditional historical mean of the factor, gives the possibility to price the different sources of risk taking into account the $p$ most recent realizations of $x_{t+1}$, and not only the most recent one, and proposes a more general term structure formula.

The plan of the paper is as follows. We consider in Section 2 the Gaussian AR $(p)$ Factor-Based Term Structure Model : the (scalar) latent factor $\left(x_{t}\right)$ driving the term structure shapes is assumed to be a gaussian $\operatorname{AR}(p)$ process, the stochastic discount factor (SDF) for the period $(t, t+1)$ is specified as an exponential-affine function of $x_{t+1}$, with a stochastic risk-correction coefficient, and the associated yield-to-maturity formula at time $t$ is an affine function of $X_{t}=\left(x_{t}, \ldots, x_{t-p+1}\right)^{\prime}$. In Section 3 we study the effect of an increasing autoregressive order $p$ on the possible shapes of term structures that the model is able to replicate, while in Section 4 we consider, under the risk-neutral measure, the Moving Average (or Heat, Jarrow and Morton) representation of the yield and short-term forward rate processes : this representation gives the possibility to exactly replicate the currently-observed yield curve. An alternative methodology to match the theoretical and the market term structure is presented in Section 5 and consists in replacing one of the model's parameter by a deterministic function of time. In Section 6 we deal with the $S$-forward framework and in Section 7 we specify the observable factor setting, where the scalar factor is the (predetermined) short rate process $\left(r_{t+1}\right)$ : we study, in particular, the problem of propagation of 
short rate shocks on the yield surface, and the problem of exact replication of the observed term structure. In Section 8 we consider the multifactor $\operatorname{VAR}(p)$ generalization [the Gaussian $\operatorname{VAR}(p)$ Factor-Based Term Structure Model], Section 9 concludes and appendices gather the proofs.

\section{Gaussian $\operatorname{AR}(p)$ Factor-Based Term Structure Models}

\subsection{Historical Dynamics}

Let us assume that the (scalar) exogenous factor $x_{t+1}$ characterizing the specification of the term structure is an $\operatorname{AR}(p)$ process of the following type:

$$
\begin{aligned}
x_{t+1} & =\nu+\varphi_{1} x_{t}+\ldots+\varphi_{p} x_{t+1-p}+\sigma \varepsilon_{t+1} \\
& =\nu+\varphi^{\prime} X_{t}+\sigma \varepsilon_{t+1},
\end{aligned}
$$

where $\varepsilon_{t+1}$ is a gaussian white noise with $\mathcal{N}(0,1)$ distribution, $\varphi=\left[\varphi_{1}, \ldots, \varphi_{p}\right]^{\prime}, X_{t}=\left[x_{t}, \ldots, x_{t+1-p}\right]^{\prime}$, and where $\sigma>0, \nu$ and $\varphi_{i}$, for $i \in\{1, \ldots, p\}$, are scalar coefficients. This model can also be represented in the following multivariate $\operatorname{AR}(1)$ form :

$$
X_{t+1}=\tilde{\nu}+\Phi X_{t}+\sigma \tilde{\varepsilon}_{t+1},
$$

where $\tilde{\nu}=[\nu, 0, \ldots, 0]^{\prime}$ and $\tilde{\varepsilon}_{t+1}=\left[\varepsilon_{t+1}, 0, \ldots, 0\right]^{\prime}$ are $p$-dimensional vectors, and where

$$
\Phi=\left[\begin{array}{cccc}
\varphi_{1} & \varphi_{2} & \ldots & \varphi_{p} \\
1 & 0 & \ldots & 0 \\
0 & 1 & \ldots & 0 \\
\vdots & \vdots & \ddots & \vdots \\
0 & \ldots & 1 & 0
\end{array}\right]
$$

is a $(p \times p)$-matrix.

\subsection{The Stochastic Discount Factor Specification}

The development of the zero-coupon bond (no arbitrage) pricing model is characterized, after the historical distribution assumption presented above, by the specification of a positive stochastic discount factor (SDF) $M_{t, t+1}$, for the period $(t, t+1)$, in order to guarantee the absence of arbitrage opportunities. The price at $t$ of a derivative paying $g\left(x_{t+H}\right)$ at $t+H$ is:

$$
\begin{aligned}
C_{t}(g, H) & =E\left[M_{t, t+1} \cdot \ldots \cdot M_{t+H-1, t+H} g\left(x_{t+H}\right) \mid I_{t}\right] \\
& =E_{t}\left[M_{t, t+H} g\left(x_{t+H}\right)\right]
\end{aligned}
$$

where $E_{t}$ denotes the expectation, under the historical probability $\mathbb{P}$, conditional on the information $I_{t}$ given by the current and the lagged values of the state variable. We choose a SDF which is exponential-affine in the state variable ${ }^{3} x_{t+1}$ :

$$
M_{t, t+1}=\exp \left[-\beta-\alpha^{\prime} X_{t}+\Gamma_{t} \varepsilon_{t+1}-\frac{1}{2} \Gamma_{t}^{2}\right],
$$

\footnotetext{
${ }^{3}$ See also Ang, Piazzesi and Wei (2006), Cochrane (2005), Gourieroux and Monfort (2006), Gourieroux, Monfort and Polimenis (2003, 2006), Gourieroux and Sufana (2003), Pegoraro (2006), Polimenis (2001).
} 
where the coefficients $\alpha=\left[\alpha_{1}, \ldots, \alpha_{p}\right]^{\prime}$ and $\beta$ are path independent, and where $\Gamma_{t}=\Gamma\left(X_{t}\right)=$ $\left(\gamma_{o}+\gamma^{\prime} X_{t}\right)$ is a stochastic risk correction coefficient which allows to well represent time variations in assets' risk premia [see sections 2.3 and 2.5]. The absence of arbitrage restriction on the discount bond with unitary residual maturity requires $E_{t}\left(M_{t, t+1}\right)=\exp \left(-r_{t+1}\right)$, where $r_{t+1}$ is the (predetermined) short-term interest rate; this condition implies the relation $r_{t+1}=\beta+\alpha^{\prime} X_{t}$. This means that, under the absence of arbitrage opportunities, the SDF can be written as:

$$
M_{t, t+1}=\exp \left[-r_{t+1}+\Gamma_{t} \varepsilon_{t+1}-\frac{1}{2} \Gamma_{t}^{2}\right]
$$

\subsection{Risk Premium}

In order to give an interpretation of the risk-correction coefficient $\Gamma_{t}$, we consider the following definition of risk premium.

Definition 1 : If we denote by $P_{t}$ the price at time $t$ of a given asset, its risk premium between $t$ and $t+1$ is :

$$
\begin{aligned}
\lambda_{t} & =\log E_{t}\left(\frac{P_{t+1}}{P_{t}}\right)-r_{t+1} \\
& =\log E_{t} \exp \left(y_{t+1}\right)-r_{t+1},
\end{aligned}
$$

where $y_{t+1}=\log \left(P_{t+1} / P_{t}\right)$ denotes the one-period geometric return of the asset.

We can interpret $\lambda_{t}$ as the excess growth rate of the expected price with respect to the present price. Now, starting from this definition of the risk premium we obtain interpretations of the function $\Gamma_{t}$, appearing in the SDF, by means of the following example.

Example : If we consider an asset providing the payoff $\exp \left(-b x_{t+1}\right)$ at $t+1$, its price in $t$ is given by:

$$
\begin{aligned}
P_{t} & =E_{t}\left[M_{t, t+1} P_{t+1}\right] \\
& =E_{t}\left[\exp \left(-r_{t+1}-\frac{1}{2} \Gamma_{t}^{2}+\left(\Gamma_{t}-b \sigma\right) \varepsilon_{t+1}-b\left(\nu+\varphi^{\prime} X_{t}\right)\right)\right] \\
& =\exp \left[-r_{t+1}-b\left(\nu+\varphi^{\prime} X_{t}\right)-b \sigma \Gamma_{t}+\frac{1}{2}(b \sigma)^{2}\right]
\end{aligned}
$$

and

$$
\begin{aligned}
E_{t} P_{t+1} & =E_{t}\left[\exp \left(-b x_{t+1}\right)\right] \\
& =\exp \left[-b\left(\nu+\varphi^{\prime} X_{t}\right)\right] E_{t}\left\{\exp \left[-b \sigma \varepsilon_{t+1}\right]\right\} \\
& =\exp \left[-b\left(\nu+\varphi^{\prime} X_{t}\right)+\frac{1}{2}(b \sigma)^{2}\right]
\end{aligned}
$$

Finally, the risk premium is:

$$
\lambda_{t}=b \sigma \Gamma_{t}
$$

Therefore, $b, \Gamma_{t}$ and $\sigma$ can be seen respectively as a risk sensitivity of the asset, a risk price and a risk measure. 


\subsection{The Affine Term Structure}

With the specification of the SDF, and applying formula (3), we determine the price of a zerocoupon bond in the following way :

$$
B(t, h)=E_{t}\left[M_{t, t+1} \cdot \ldots \cdot M_{t+h-1, t+h}\right]
$$

where $B(t, h)$ denoted the price at time $t$ for a time to maturity $h$.

Note that, for arbitrary real constants $\mu_{1}$ and $\mu_{2}$, we obtained the same SDF dynamics if we replace $x_{t}$ by $\mu_{1}+\mu_{2} x_{t}$ (and therefore $X_{t}$ by $\mu_{1} e+\mu_{2} X_{t}$, with $e=(1, \ldots, 1)^{\prime} \in \mathbb{R}^{p}$ ), $\gamma$ by $\frac{\gamma}{\mu_{2}}, \gamma_{o}$ by $\gamma_{o}-\frac{\mu_{1}}{\mu_{2}} \gamma^{\prime} e, \alpha$ by $\frac{\alpha}{\mu_{2}}$ and $\beta$ by $\beta-\frac{\mu_{1}}{\mu_{2}} \alpha^{\prime} e$. Therefore, if $x_{t}$ is not directly observed, we can assume for instance, as far as the term structure is concerned, that $\nu=0$ and $\sigma=1$, or $\beta=0$ and $\alpha_{1}=1$.

Proposition 1 : The price at date $t$ of the zero-coupon bond with time to maturity $h$ is :

$$
B(t, h)=\exp \left(c_{h}^{\prime} X_{t}+d_{h}\right),
$$

where $c_{h}$ and $d_{h}$ satisfies the recursive equations :

$$
\begin{aligned}
c_{h} & =-\alpha+\Phi^{\prime} c_{h-1}+c_{1, h-1} \sigma \gamma \\
& =-\alpha+\Phi^{*^{\prime}} c_{h-1}, \\
d_{h} & =-\beta+c_{1, h-1}\left(\nu+\gamma_{o} \sigma\right)+\frac{1}{2} c_{1, h-1}^{2} \sigma^{2}+d_{h-1},
\end{aligned}
$$

with :

$$
\Phi^{*}=\left[\begin{array}{cccc}
\varphi_{1}+\sigma \gamma_{1} & \varphi_{2}+\sigma \gamma_{2} & \ldots & \varphi_{p}+\sigma \gamma_{p} \\
1 & 0 & \ldots & 0 \\
0 & 1 & \ldots & 0 \\
\vdots & \vdots & \ddots & \vdots \\
0 & \ldots & 1 & 0
\end{array}\right]
$$

and where $h \geq 1$, with initial conditions $c_{0}=0, d_{0}=0$ (or $c_{1}=-\alpha, d_{1}=-\beta$ ); $c_{1, h}$ is the first component of the $p$-dimensional vector $c_{h}$. [Proof : see Appendix 1.]

Corollary 1 : The yields to maturity associated to formula (9) are :

$$
\begin{aligned}
R(t, h) & =-\frac{1}{h} \log B(t, h) \\
& =-\frac{c_{h}^{\prime}}{h} X_{t}-\frac{d_{h}}{h}, \quad h \text { varying, }
\end{aligned}
$$

and they are affine functions of the factor $X_{t}$, that is of the $p$ most recent lagged values of $x_{t+1}$.

\subsection{Excess Returns of Zero-Coupon Bonds}

In our framework (with $B^{*}(t, T)=B(t, T-t)$ ), we have the following specification for the zerocoupon bond return process. 
Proposition 2 : Under the absence of arbitrage opportunity, and for a fixed maturity $T$, the oneperiod geometric zero-coupon bond return process $\rho=[\rho(t, T), 0 \leq t \leq T]$, where $\rho(t+1, T)=$ $\log \left[B^{*}(t+1, T)\right]-\log \left[B^{*}(t, T)\right]$, is given by:

$$
\rho(t+1, T)=r_{t+1}-\frac{1}{2} \omega(t+1, T)^{2}+\omega(t+1, T) \Gamma_{t}-\omega(t+1, T) \varepsilon_{t+1},
$$

where $\omega(t+1, T)=-\left(\sigma c_{1, T-t-1}\right)$ [Proof : see Appendix 2].

This means that the process $\rho$ is such that, conditionally to $\underline{x_{t}}, \rho(t+1, T)$ is normally distributed with mean $\mu(t+1, T)=r_{t+1}-\frac{1}{2} \omega(t+1, T)^{2}+\omega(t+1, T) \Gamma_{t}$ and variance $\omega(t+1, T)^{2}=\left(\sigma c_{1, T-t-1}\right)^{2}$.

The associated risk premium between $t$ and $t+1$, denoted by $\lambda_{t}(T)$, is:

$$
\lambda_{t}(T)=\log E_{t} \exp [\rho(t+1, T)]-r_{t+1}=\omega(t+1, T) \Gamma_{t} .
$$

We note that, $\Gamma_{t}=\left(\gamma_{o}+\gamma^{\prime} X_{t}\right)$ plays for any $T$ the role of a risk premium per unit of "risk" $\omega(t+1, T)$ : in particular, the variability of $\lambda_{t}(T)$ is driven, for a fixed $\gamma$ different from zero, by the $p$ most recent lagged values of $x_{t+1}$. If we assume $\gamma=0$ (i. e., $\Gamma_{t}=\gamma_{o}$ ), the risk correction coefficient and the risk premium of the zero-coupon bond become constants. Also note that, if $T=t+2$ and $x_{t}=r_{t+1}$, we have $\omega(t+1, T)=\sigma$ and we get the result of the example presented in section 2.3 for $b=1$.

\subsection{Risk-Neutral Dynamics}

In the previous sections we have presented the Gaussian $\operatorname{AR}(p)$ Term Structure model under the historical probability $\mathbb{P}$. Now, it is well known from asset pricing theory that, under the absence of arbitrage opportunity, there exist equivalent (to $\mathbb{P}$ ) probability measures under which asset prices, evaluated with respect to some numeraire ${ }^{4} N_{t}$, are martingales. This change of measure is important in an asset pricing perspective if it leads to convenient closed-form or numerically tractable pricing formulas. The most used choices of numeraire are the money-market account, presented in this section, and the zero-coupon bond choice, presented in the following section.

If we consider as numeraire the money-market account $N_{t}=\exp \left(r_{1}+\ldots+r_{t}\right)=\left(A_{0, t}\right)^{-1}$, where $A_{0, t}=E_{0}\left(M_{0,1}\right) \cdots E_{t-1}\left(M_{t-1, t}\right)$, the associated equivalent probability $\mathbb{Q}_{t}$ has a one-period conditional (to $I_{t}$ ) density, with respect to $\mathbb{P}_{t}$, given by :

$$
\frac{d \mathbb{Q}_{t}}{d \mathbb{P}_{t}}=\frac{A_{0, t} M_{t, t+1}}{A_{0, t+1}}=\frac{M_{t, t+1}}{E_{t}\left(M_{t, t+1}\right)} .
$$

In a general $(T-t)$-period horizon, the conditional (to $I_{t}$ ) density of the risk-neutral probability $\mathbb{Q}_{t}^{T}$ with respect to the historical probability $\mathbb{P}_{t}^{T}$ is given by :

$$
\frac{d \mathbb{Q}_{t}^{T}}{d \mathbb{P}_{t}^{T}}=\frac{M_{t, t+1} \cdot \ldots \cdot M_{T-1, T}}{E_{t}\left(M_{t, t+1}\right) \cdot \ldots \cdot E_{T-1}\left(M_{T-1, T}\right)},
$$

and the associated risk-neutral pricing formula for a derivative paying $g\left(x_{T}\right)$ at $T$ is:

$$
\begin{aligned}
C^{*}(t, T) & =E_{t}^{\mathbb{Q}}\left[E_{t}\left(M_{t, t+1}\right) \cdot \ldots \cdot E_{T-1}\left(M_{T-1, T}\right) g\left(x_{T}\right)\right] \\
& =E_{t}^{\mathbb{Q}}\left[\exp \left(-r_{t+1}-\ldots-r_{T}\right) g\left(x_{T}\right)\right] .
\end{aligned}
$$

\footnotetext{
${ }^{4} \mathrm{~A}$ numeraire is defined as a non-dividend-paying price process $N=\left(N_{t}, t \geq 0\right)$ with $N_{0}=1$; in other words, $N$ is a stochastic process such that, for every $T>t, N_{t}=E_{t}\left[M_{t, T} N_{T}\right]$ and $E_{0}\left[M_{0, T} N_{T}\right]=1$, where $M_{t, T}=$ $M_{t, t+1} \cdot \ldots \cdot M_{T-1, T}$. The process $N^{*}=\left(N_{t} M_{0, t}, t \geq 0\right)$ is therefore a $\mathbb{P}$-martingale with unitary value in $t=0$, and if $\mathbb{Q}$ is the probability defined by the sequence of conditional densities $\frac{N_{t+1} M_{t, t+1}}{N_{t}}$ with respect to $\mathbb{P}$, a price process $S_{t}$ is such that $S_{t} / N_{t}$ is a $\mathbb{Q}$-martingale.
} 
The one-period transition from the historical world to the risk-neutral one is given, in our framework, by the conditional density function :

$$
\frac{M_{t, t+1}}{E_{t}\left(M_{t, t+1}\right)}=\exp \left[\Gamma_{t} \varepsilon_{t+1}-\frac{1}{2} \Gamma_{t}^{2}\right] .
$$

Moreover, for any asset, the price $P_{t}$ at $t$ is equal to $\exp \left(-r_{t+1}\right) E_{t}^{\mathbb{Q}}\left(P_{t+1}\right)$ and, therefore, the risk premium $\lambda_{t}$ presented in Definition 1 is equal to $\log E_{t}\left(P_{t+1}\right)-\log E_{t}^{\mathbb{Q}}\left(P_{t+1}\right)$.

The risk-neutral Laplace transform of $x_{t+1}$, conditionally to $\underline{x_{t}}$, is given by:

$$
\begin{aligned}
& E_{t}^{\mathbb{Q}}\left[\exp \left(u x_{t+1}\right)\right]= E_{t}\left[\frac{M_{t, t+1}}{E_{t}\left(M_{t, t+1}\right)} \exp \left(u x_{t+1}\right)\right] \\
&= E_{t}\left[\exp \left(\left(\gamma_{o}+\gamma^{\prime} X_{t}\right) \varepsilon_{t+1}-\frac{1}{2}\left(\gamma_{o}+\gamma^{\prime} X_{t}\right)^{2}+u x_{t+1}\right)\right] \\
&= \exp \left[u\left(\nu+\varphi^{\prime} X_{t}\right)-\frac{1}{2}\left(\gamma_{o}+\gamma^{\prime} X_{t}\right)^{2}\right] \\
& E_{t}\left[\exp \left(\gamma_{o}+\gamma^{\prime} X_{t}+u \sigma\right) \varepsilon_{t+1}\right] \\
&=\exp \left[u\left[\left(\nu+\sigma \gamma_{o}\right)+(\varphi+\sigma \gamma)^{\prime} X_{t}\right]+\frac{1}{2} u^{2} \sigma^{2}\right],
\end{aligned}
$$

where $\varphi=\left[\varphi_{1}, \ldots, \varphi_{p}\right]^{\prime}$. Therefore, we get the following result.

Proposition 3 : Under the risk-neutral probability $\mathbb{Q}, x_{t+1}$ is an $\operatorname{AR}(p)$ process of the following type:

$$
x_{t+1}=\nu^{*}+\varphi_{1}^{*} x_{t}+\ldots+\varphi_{p}^{*} x_{t+1-p}+\sigma^{*} \eta_{t+1}
$$

with

$$
\begin{aligned}
\nu^{*} & =\left(\nu+\sigma \gamma_{o}\right) \\
\varphi_{i}^{*} & =\left(\varphi_{i}+\sigma \gamma_{i}\right) \quad \text { for } i \in\{1, \ldots, p\} \\
\sigma^{*} & =\sigma,
\end{aligned}
$$

where $\eta_{t+1} \stackrel{\mathbb{Q}}{\sim} \mathcal{I} \mathcal{I N}(0,1)$. Note that $\varepsilon_{t+1}=\eta_{t+1}+\Gamma_{t}$.

This model can be represented in the following vectorial form :

$$
X_{t+1}=\tilde{\nu}^{*}+\Phi^{*} X_{t}+\sigma^{*} \tilde{\eta}_{t+1},
$$

where $\tilde{\nu}^{*}=\left[\nu^{*}, 0, \ldots, 0\right]^{\prime}$ and $\tilde{\eta}_{t+1}=\left[\eta_{t+1}, 0, \ldots, 0\right]^{\prime}$ are $p$-dimensional vectors, and where $\Phi^{*}$ has been introduced in Section 2.4.

We observe that, given the stochastic specification of the risk-premium, in the risk-neutral world $x_{t+1}$ has not only a different constant term, but also different autoregressive coefficients.

With regard to the zero-coupon bond return process, under the risk-neutral probability we have the following specification.

Proposition 4 : In the risk-neutral framework, for a fixed maturity $T$, the one-period geometric zero-coupon bond return process satisfies the relation:

$$
\rho(t+1, T)=r_{t+1}-\frac{1}{2} \omega(t+1, T)^{2}-\omega(t+1, T) \eta_{t+1},
$$


with a risk premium equal to :

$$
\lambda_{t}^{\mathbb{Q}}(\rho, 1)=\log E_{t}^{\mathbb{Q}} \exp [\rho(t+1, T)]-r_{t+1}=0 .
$$

[Proof : see Appendix 3].

\section{Term Structure Shapes}

\subsection{General Results}

The different shapes that the yield curve relation (11) is able to reproduce depend crucially on the system of difference equations (10). Taking into account the result presented in Proposition 1, the system of linear difference equations characterizing $\left(c_{h}, d_{h}\right)$, for $h \geq 1$, can be written as:

$$
\left\{\begin{array}{l}
c_{h}=\Phi^{*^{\prime}} c_{h-1}-\alpha \\
d_{h}=-\beta+c_{1, h-1} \nu^{*}+\frac{1}{2} c_{1, h-1}^{2} \sigma^{2}+d_{h-1}
\end{array}\right.
$$

with initial conditions $c_{0}=0$ and $d_{0}=0$; in this case, it is well known that the steady state $\overline{\mathbf{C}}=\left[\bar{c}_{1}, \ldots, \bar{c}_{p}\right]^{\prime}$ of the system $c_{h}$ is given, $I$ denoting the $(p \times p)$ identity matrix, by:

$$
\overline{\mathbf{C}}=-\left(I-\Phi^{*^{\prime}}\right)^{-1} \alpha,
$$

under the (stability) condition that the $p$ eigenvalues $\left(\lambda_{1}, \ldots, \lambda_{p}\right)$ of $\Phi^{*^{\prime}}$ are all smaller than unity in modulus, or, equivalently, that the risk-neutral dynamics of $x_{t}$ is stationary, or that the roots of the risk-neutral autoregressive polynomial (of degree $p$ ) $\Psi^{*}(L)=1-\varphi_{1}^{*} L-\ldots-\varphi_{p}^{*} L^{p}$ have a modulus larger than one (given that these roots are the inverse of the eigenvalues). More precisely, the system of equations $c_{h}$ can be rewritten as:

$$
\left\{\begin{aligned}
c_{1, h} & =\varphi_{1}^{*} c_{1, h-1}+c_{2, h-1}-\alpha_{1} \\
c_{2, h} & =\varphi_{2}^{*} c_{1, h-1}+c_{3, h-1}-\alpha_{2} \\
& \vdots \\
c_{p-1, h} & =\varphi_{p-1}^{*} c_{1, h-1}+c_{p, h-1}-\alpha_{p-1} \\
c_{p, h} & =\varphi_{p}^{*} c_{1, h-1}-\alpha_{p},
\end{aligned}\right.
$$

and if we substitute the $p^{t h}$ equation in the $(p-1)^{t h}$ for $c_{p, h-1}$, and then the $(p-1)^{t h}$ equation in the $(p-2)^{t h}$ for $c_{p-1, h-1}$, and so on till the first one, we find that $c_{1, h}$ is described by the following $p^{\text {th }}$ order linear difference equation :

$$
\Psi^{*}(L) c_{1, h}=-\sum_{i=1}^{p} \alpha_{i}
$$

where $\Psi^{*}(L)=1-\varphi_{1}^{*} L-\ldots-\varphi_{p}^{*} L^{p}$ operates here to $h$. The remaining equations are given by :

$$
c_{p-j, h}=-\sum_{i=0}^{j} \alpha_{p-i}+\sum_{i=0}^{j} \varphi_{p-i}^{*} c_{1, h-j+i-1}, \quad j \in\{0, \ldots, p-2\} .
$$


Given the risk-neutral stationary assumption on the $x_{t}$ process, the $c_{p-j, h}$, for $j \in\{0, \ldots, p-1\}$, converge at an exponential rate with possible oscillations, when $h \rightarrow \infty$. The limits are :

$$
\begin{aligned}
\bar{c}_{1} & =-\frac{\sum_{i=1}^{p} \alpha_{i}}{\Psi^{*}(1)} \\
\bar{c}_{p-j} & =-\sum_{i=0}^{j} \alpha_{p-i}+\bar{c}_{1} \sum_{i=0}^{j} \varphi_{p-i}^{*}, \quad j \in\{0, \ldots, p-2\} ;
\end{aligned}
$$

note that $\Psi^{*}(1)>0$ because of the stability conditions. In the observable factor framework with $x_{t}=r_{t+1}, \alpha=e_{1}$ and $\beta=0$ because of the absence of arbitrage restrictions [see Section 7.1], we have $\bar{c}_{1}=-\Psi^{*}(1)^{-1}<0$; in the latent factor setting we can always assume $\sum_{i=1}^{p} \alpha_{i}>0$ and therefore $\bar{c}_{1}<0$ also.

With regard to $d_{h}$, its equation gives the specification of the long-term yield $R(t, \infty)$ as a function of the steady state $\bar{c}_{1}$; indeed, the difference equation $d_{h}$ can be written (assuming the identification condition $\beta=0$ ) as:

$$
d_{h}= \begin{cases}0 & \text { for } h=1, \\ \nu^{*} \sum_{j=1}^{h-1} c_{1, j}+\frac{1}{2} \sigma^{2} \sum_{j=1}^{h-1} c_{1, j}^{2}, & \forall h \geq 2,\end{cases}
$$

and, under the stability of the system $c_{h}$, we have from relation (11) that:

$$
\begin{aligned}
R(t, \infty) & =\lim _{h \rightarrow+\infty} R(t, h) \\
& =\lim _{h \rightarrow+\infty}-\frac{c_{h}{ }^{\prime}}{h} X_{t}-\frac{\nu^{*}}{h} \sum_{j=1}^{h-1} c_{1, j}-\frac{\sigma^{2}}{2 h} \sum_{j=1}^{h-1} c_{1, j}^{2}=-\bar{c}_{1} \nu^{*}-\frac{1}{2}\left(\bar{c}_{1} \sigma\right)^{2},
\end{aligned}
$$

which is positive under the condition $\left[\nu^{*}+\frac{1}{2} \sigma^{2} \bar{c}_{1}\right]>0$.

The shape of the $c_{h}$, for $h$ varying, depends on whether the eigenvalues $\left(\lambda_{1}, \ldots, \lambda_{p}\right)$ of $\Phi^{*^{\prime}}$ are real or complex, single or multiple, larger or smaller than one in modulus.

The purpose of the following examples is to study the (quantitative and qualitative) properties of (10) and to represent, with some numerical examples, the associated possible shapes of the term structures : here, the values of the parameters are initially fixed on the basis of estimation results presented in Monfort and Pegoraro (2006), and then, variations on each parameter are applied in order to study the richness of shapes the models are able to replicate. Now, given that for the parameters' estimation Monfort and Pegoraro (2006) consider as short rate the yield with time to maturity equal to one month $\left[r_{t+1}=R(t, 1)\right]$, the parameter values are expressed (along with the short rate itself) on a monthly basis.

Let us study more deeply the solutions of $c_{h}$ and $d_{h}$ in the case of $p=1$ [Section 3.2.1] and $p=2$ [Section 3.2.2], and the term structure shapes the $\operatorname{AR}(p)$ Factor-Based Models are able to replicate when $p \in\{1, \ldots, 4\}$. 


\subsection{Gaussian AR $(p)$ Factor-Based Term Structure Shapes}

\subsubsection{The case $p=1$}

When the scalar observable factor $x_{t}=r_{t+1}$ follows a Gaussian AR(1) process, the associated term structure model is the discrete-time equivalent of the Vasicek model, with a stochastic risk premium. In this case $c_{h}$ satisfies the fist-order difference equation:

$$
c_{h}=-1+(\varphi+\sigma \gamma) c_{h-1},
$$

where $\sigma>0, \gamma$ and $|\varphi|<1$ are scalar coefficients, and with a general solution, denoted $c(h)$, given by:

$$
c(h)=-\left[\frac{1}{1-(\varphi+\sigma \gamma)}\right]\left[1-(\varphi+\sigma \gamma)^{h}\right]=-\left[\frac{1-\varphi^{* h}}{1-\varphi^{*}}\right],
$$

which tends, for $h$ increasing to infinity, to the limit:

$$
\bar{c}=-\left[\frac{1}{1-\varphi^{*}}\right]
$$

under the condition $\left|\varphi^{*}\right|<1$, where $\varphi^{*}=(\varphi+\sigma \gamma)$ is the unique eigenvalue of the (scalar) matrix $\Phi^{*^{\prime}}$; this condition implies $c(h)<0$ for every $h>0$. In addition, if $0<\varphi+\sigma \gamma<1$ (respectively, $-1<\varphi+\sigma \gamma<0$ ), the function $c(h)$ converges in decreasing (respectively, oscillating) towards $\bar{c}$. With regard to $d_{h}$, it easy to verify that :

$$
\begin{gathered}
d(h)=-\left[\frac{\nu^{*}}{1-\varphi^{*}}\right](h-1)+\left[\frac{\varphi^{*}-\varphi^{* h}}{1-\varphi^{*}}\right]\left[\frac{\nu^{*}}{1-\varphi^{*}}-\frac{\sigma^{2}}{\left(1-\varphi^{*}\right)^{2}}\right] \\
+\frac{\sigma^{2}}{2\left(1-\varphi^{*}\right)^{2}}\left[(h-1)+\frac{\varphi^{* 2}-\varphi^{* 2 h}}{1-\varphi^{* 2}}\right] ;
\end{gathered}
$$

consequently, the yield to maturity formula (11), for $p=1$, is given by :

$$
\begin{gathered}
R(t, h)=\frac{1}{h}\left[\frac{1-\varphi^{* h}}{1-\varphi^{*}}\right] r_{t+1}+\frac{(h-1)}{h}\left[\frac{\nu^{*}}{1-\varphi^{*}}\right]-\frac{1}{h}\left[\frac{\varphi^{*}-\varphi^{* h}}{1-\varphi^{*}}\right]\left[\frac{\nu^{*}}{1-\varphi^{*}}-\frac{\sigma^{2}}{\left(1-\varphi^{*}\right)^{2}}\right] \\
-\frac{\sigma^{2}}{2 h\left(1-\varphi^{*}\right)^{2}}\left[(h-1)+\frac{\varphi^{* 2}-\varphi^{* 2 h}}{1-\varphi^{* 2}}\right] .
\end{gathered}
$$

Observe that, in the classical continuous-time Vasicek model, the market risk premium is constant $(\gamma=0)$.

Examples of the term structures are provided in Figures 1 to 4 . For a value of $\varphi^{*}=0.99$, $r_{t+1}=0.003$ and $\sigma^{2}=0.00000039$, we observe in Figure 1 that a value of $\nu^{*}$ increasing from 0.00005 to 0.00030 induce the term structure to move from an almost flat shape to a monotone increasing one. In Figure 2 we study once more the effect of variations in $\nu^{*}$ on the term structure with $\varphi^{*}=0.99$ and $r_{t+1}=0.003$, but now we fix $\sigma^{2}=0.000008$ and $\nu^{*}$ increases from 0.00010 to 0.00015 : in this case the yield curve became humpshaped. In Figure 3 we observe the effect on the yield curve of a value of $\varphi^{*}$ increasing from 0.87 to 0.99 , with $\varphi=0.95, r_{t+1}=0.003$, $\sigma^{2}=0.00000039$ and $\nu^{*}=0.00007$ : starting from a monotone decreasing shape, the yield curve find the Vasicek case for $\varphi^{*}=\varphi=0.95$ (dashed line) and ends with a monotone increasing shape 
for $\varphi^{*}=0.99$. Figure 4 presents the effect on the term structure of $\sigma^{2}$ increasing from 0.0000004 to 0.0000024: the shape moves from a monotone increasing case to a humped one.

These numerical examples show the shapes the classical one-factor, Markovian of order one, term structure models [Vasicek (1977), Cox, Ingersoll and Ross (1985), Pearson and Sun (1994)] are able to reproduce: monotone increasing, monotone decreasing, flat and humpshaped term structures [see Figure A]. Instead, we observe also yield curves with different shapes like, for instance, $J$-shaped (when the yield curve has an interior minimum), $L$-shaped (when the yield curve, starting from $r_{t+1}$ at $h=1$, takes a decidedly lower value at the following maturities, and then it remains at an almost constant level), inverted $L$-shaped (when the yield curve, starting from $r_{t+1}$ at $h=1$, takes a decidedly higher value at the following maturities, and then it remains at an almost constant level) or $J$-humpshaped (when the yield curve has, first, an interior minimum, and then an interior maximum) term structures [see Figures B, C and D].

The scalar Gaussian AR $(p)$ Factor-Based Term Structure model, for $p>1$, is able to overcome these limits and, in particular is able to replicate the observed term structures presented in Figures $\mathrm{B}, \mathrm{C}$ and D. We present here below, the Gaussian AR(2) case and we give examples of yield curves for $p \in\{2,3,4\}$.

\subsubsection{The case $p=2$}

If the factor $x_{t}=r_{t+1}$ is a Gaussian $\operatorname{AR}(2)$ process, the recursive equation for $c_{h}$ is described by a first-order $(2 \times 2)$ system of difference equations of the following type:

$$
\left[\begin{array}{l}
c_{1, h} \\
c_{2, h}
\end{array}\right]-\left[\begin{array}{ll}
\varphi_{1}+\sigma \gamma_{1} & 1 \\
\varphi_{2}+\sigma \gamma_{2} & 0
\end{array}\right]\left[\begin{array}{l}
c_{1, h-1} \\
c_{2, h-1}
\end{array}\right]=-\left[\begin{array}{l}
1 \\
0
\end{array}\right]
$$

substituting the first equation into the second, we find for $c_{1, h+1}$ the following second-order linear difference equation:

$$
c_{1, h+1}=-1+\varphi_{1}^{*} c_{1, h}+\varphi_{2}^{*} c_{1, h-1},
$$

where $\varphi_{1}^{*}=\left(\varphi_{1}+\sigma \gamma_{1}\right)$ and $\varphi_{2}^{*}=\left(\varphi_{1}+\sigma \gamma_{2}\right)$; under the condition that the two eigenvalues $\left(\lambda_{1}, \lambda_{2}\right)$ of $\Phi^{*^{\prime}}$ (or the inverse of the roots of $1-\varphi_{1}^{*} L-\varphi_{2}^{*} L^{2}$ ) are not equal and less than unity in modulus, and regardless of their real or complex nature, the limit of $c_{1, h}$ is given by:

$$
\bar{c}_{1}=-\frac{1}{\left(1-\lambda_{1}\right)\left(1-\lambda_{2}\right)}
$$

these conditions can equivalently be expressed in the following way : $\varphi_{1}^{*}+\varphi_{2}^{*}<1, \varphi_{2}^{*}-\varphi_{1}^{*}<1$ and $\left|\varphi_{2}^{*}\right|<1$. If we substitute $\bar{c}_{1}$ into the second equation of system (23) we find, consequently, the limit of $c_{2, h}$ :

$$
\bar{c}_{2}=-\varphi_{2}^{*} \frac{1}{\left(1-\lambda_{1}\right)\left(1-\lambda_{2}\right)} .
$$

The recursive equation characterizing $d_{h}$ is given by $(22)$.

Examples of the yield curves that the Gaussian $\operatorname{AR}(2)$ model is able to replicate are presented in Figures 5 to 8 . In Figure 5 we consider $\varphi_{1}^{*}=0.74, \varphi_{2}^{*}=0.24, \sigma^{2}=0.00000039$, with $r_{t+1}=0.0036$ and $r_{t}=0.0030$, and we observe what happens when $\nu^{*}$ increases from 0.00005 to 0.00030 ; the curve start from an $L$-shape and then, as $\nu^{*}$ increases, the long-term yield increases towards larger values with the term structure taking a $J$-shape. In Figure 6 we study what happens when $\varphi_{2}^{*}$ 
increases from 0.04 to 0.24 , with $\varphi_{1}^{*}=0.74, \nu^{*}=0.00008, \sigma^{2}=0.00000039$, and with $r_{t+1}=0.0030$ and $r_{t}=0.0036$. The term structure is initially humpshaped, with an interior maximum for a short maturity ( $h=2$ months), and with a long rate much lower than the short rate; then, as $\varphi_{2}^{*}$ increases, the long rate increases till levels larger than the short rate; here, for $\varphi_{2}^{*}=0.24$, the curve takes an inverted $L$-shape.

In Figure 7 we fix $\varphi_{1}^{*}=0.74, \varphi_{2}^{*}=0.24, \sigma^{2}=0.000008$, with $r_{t+1}=0.0032$ and $r_{t}=0.0036$, and we study the effect of $\nu^{*}$ increasing from 0.00010 to 0.00015 : the curves are humpshaped as in Figure 2 [Gaussian $\operatorname{AR}(1)$ case], but now we have a strong increment in the yield levels when we move from $h=1$ to $h=3$. In Figure 8 we have $\varphi_{1}^{*}=0.74, \varphi_{2}^{*}=0.24$ and $\nu^{*}=0.00007$, with $r_{t+1}=0.0036$ and $r_{t}=0.0032$; we study the effect on the term structure of $\sigma^{2}$ increasing from 0.0000004 to 0.0000024 : the curve is always $J$-shaped, with an interior minimum for $h=2$, but when $\sigma^{2}$ increases, the long rate $(h=60$ months $)$ moves from values larger to values lower than the $r_{t+1}$, and a hump forms at intermediate maturity yields.

\subsubsection{Term Structure Shapes for $p=3$ and $p=4$}

In Figures 9 to 12 we present the yield curves associated to the Gaussian AR(3) specification: we observe that this model is able to replicate the same kind of shapes as the Gaussian AR(2) case, but with curves which are smoother in the short term part as we frequently observe [see the inverted $L$-shaped curves in Figures C and D].

The Gaussian AR(4) case further enrich, with respect to the previous cases, the family of term structure shapes [see Figures 13 to 16] : more precisely, this model is able to replicate term structure with two interior local maxima and an interior local minimum [see Figure 14], or two interior local minima and an two interior local maxima [see Figure 15] concentrated at short maturities, or yield curves with several changes in the slope around short maturities [see Figures 13 and 16].

The examples presented above show that our (discrete-time) multi-lag approach gives the possibility to reproduce, with a scalar factor, yield curves that Markovian of order one univariate models are not able to replicate. In particular, we have see that the introduction of several lags allow the model to reproduce $J$-shaped, $L$-shaped, inverted $L$-shaped and $J$-humpshaped yield curves we observe on the data.

\section{Moving Average or HJM Representations}

\subsection{Risk-Neutral Moving Average Representation of the Yield Process}

The purpose of this section is to derive the joint risk-neutral dynamics of the yield to maturity processes $R^{*}(\cdot, T)=\left[R^{*}(t, T), 0 \leq t \leq T\right]$ (with $R^{*}(t, T)=R(t, T-t)$ ), driven by the factor $\left(x_{t}\right)$ introduced above, conditionally to the initial market yield curve $R_{M}^{*}=\left\{R_{M}^{*}(0, \tau), \tau \geq 0\right\}$.

More precisely, we start in this section with the derivation of the joint risk-neutral dynamics of the processes $\left\{R^{*}(\cdot, T)\right\}$, based on the representation of the log-price zero-coupon bond process in terms of the conditional variances of the bond return process $\rho(t, T)$. In the next section we will represent the process $R^{*}(\cdot, T)$ in terms of the forward-rate volatility structure.

Starting from the identity

$$
\log \left[B^{*}(t, T)\right]=\sum_{j=1}^{t} \rho(j, T)+\log \left[B^{*}(0, T)\right],
$$

we have in the risk-neutral world, using (19):

$$
\log \left[B^{*}(t, T)\right]=-\sum_{j=1}^{t} \omega(j, T) \eta_{j}+\sum_{j=1}^{t} r_{j}-\frac{1}{2} \sum_{j=1}^{t} \omega(j, T)^{2}+\log \left[B^{*}(0, T)\right],
$$


and, consequently, we find :

Proposition 5 : For every fixed maturity $T$, the zero-coupon bond price process $B^{*}(\cdot, T)=$ $\left[B^{*}(t, T), 0 \leq t \leq T\right]$, under the risk-neutral probability $\mathbb{Q}$, has the following representation:

$$
B^{*}(t, T)=B^{*}(0, T) \exp \left[\sum_{j=1}^{t}\left[r_{j}-\frac{1}{2} \omega(j, T)^{2}\right]-\sum_{j=1}^{t} \omega(j, T) \eta_{j}\right],
$$

where $\eta_{j} \stackrel{\mathbb{Q}}{\sim} \mathcal{I} \mathcal{I} \mathcal{N}(0,1)$, with $j \in\{1, \ldots, t\}$.

If we put $T=t$ in (26), we get a relation for the sum of the short-rates:

$$
\sum_{j=1}^{t} r_{j}=\sum_{j=1}^{t} \omega(j, t) \eta_{j}+\frac{1}{2} \sum_{j=1}^{t} \omega(j, t)^{2}-\log \left[B^{*}(0, t)\right]
$$

that we can substitute in (26) to find the following alternative representation for the bond price process :

Proposition 6 : For every fixed maturity $T$, the zero-coupon bond price process $B^{*}(\cdot, T)=$ $\left[B^{*}(t, T), 0 \leq t \leq T\right]$, under the risk-neutral probability $\mathbb{Q}$, can be written as :

$$
B^{*}(t, T)=\frac{B^{*}(0, T)}{B^{*}(0, t)} \exp \left(-\sum_{j=1}^{t}[\omega(j, T)-\omega(j, t)] \eta_{j}-\frac{1}{2} \sum_{j=1}^{t}\left[\omega(j, T)^{2}-\omega(j, t)^{2}\right]\right) .
$$

Relation (29) leads to the following proposition:

Proposition 7 : For every fixed maturity $T$, the yield process $R^{*}(\cdot, T)=\left[R^{*}(t, T), 0 \leq t \leq T\right]$ has, under the risk-neutral probability $\mathbb{Q}$, the following representation:

$$
\begin{gathered}
R^{*}(t, T)=-\frac{1}{T-t} \log \left[\frac{B^{*}(0, T)}{B^{*}(0, t)}\right]+\frac{1}{T-t} \sum_{j=1}^{t}[\omega(j, T)-\omega(j, t)] \eta_{j} \\
+\frac{1}{T-t} \frac{1}{2} \sum_{j=1}^{t}\left[\omega(j, T)^{2}-\omega(j, t)^{2}\right]
\end{gathered}
$$

Conditionally to the information $\underline{x_{0}}$, and for every maturity $T$, the processes $\left\{R^{*}(\cdot, T)\right\}$ are, under the risk-neutral probability $\mathbb{Q}$, joint $\mathrm{MA}$ processes with time-varying coefficients, driven by the same white noise $\eta_{t}$, and the past information appears through the term structure at date $t=0$.

If we identify the term structure at date $t=0$ with the market yield curve $R_{M}^{*}$, the yield process $R^{*}(\cdot, T)$ exactly replicates the term structure observed at the current time $t=0$. We will see in Section 5 a different approach able to guarantee the exact fitting of $R_{M}^{*}$.

\subsection{The Risk-Neutral MA Representation of Short-Term Forward Rates: the HJM Framework}

It is possible to translate the results we have presented above in terms of short-term forward rates as in the Heath, Jarrow and Morton (1992) (henceforth HJM) approach ${ }^{5}$. In particular, if we denote the one-period forward rate as $f(t, T)=\log \left[B^{*}(t, T)\right]-\log \left[B^{*}(t, T+1)\right]$, and if we use relation (29) we have :

\footnotetext{
${ }^{5}$ In their paper, which generalizes the discrete-time Ho and Lee (1986) model, HJM (1992) proposed the instantaneous forward rate as the factor to model, and, under the absence of arbitrage, they derived the stochastic evolution of the yield curve, with the forward-rates dynamics fully specified by their instantaneous volatility structures.
} 
Proposition 8 : For any fixed maturity $T$, the forward rate process $f(t, T)$ satisfies, under the risk-neutral measure:

$$
f(t, T)=f(0, T)+\sum_{j=1}^{t}[\omega(j, T+1)-\omega(j, T)] \eta_{j}+\frac{1}{2} \sum_{j=1}^{t}\left[\omega(j, T+1)^{2}-\omega(j, T)^{2}\right],
$$

where $f(0, T)=\log \left[\frac{B^{*}(0, T)}{B^{*}(0, T+1)}\right]$.

Proposition 9 : The one-period forward rate increment $\Delta f(t, T)=f(t+1, T)-f(t, T)$ satisfies:

$$
\Delta f(t, T)=\mu^{\mathbb{Q}}(t+1, T)+\sigma^{\mathbb{Q}}(t+1, T) \eta_{t+1},
$$

where

$$
\begin{aligned}
\sigma^{\mathbb{Q}}(t+1, T) & =\omega(t+1, T+1)-\omega(t+1, T) \\
\mu^{\mathbb{Q}}(t+1, T) & =\frac{1}{2}\left[\omega(t+1, T+1)^{2}-\omega(t+1, T)^{2}\right] \\
& =\sigma^{\mathbb{Q}}(t+1, T)\left[\frac{\sum_{\tau=t+1}^{T} \sigma^{\mathbb{Q}}(t+1, \tau)+\sum_{\tau=t+1}^{T-1} \sigma^{\mathbb{Q}}(t+1, \tau)}{2}\right] .
\end{aligned}
$$

[Proof : see Appendix 4].

For every maturity $T$, the process $f(\cdot, T)=[f(t, T), 0 \leq t \leq T]$ has, therefore, risk-neutral independent increments and a dynamics fully specified by the conditional volatilities of these increments. Relation (31) can be rewritten in the following way:

Corollary 2 : For any fixed maturity $T$, the forward rate $f(t, T)$ risk-neutral dynamics is given by:

$$
f(t, T)=f(0, T)+\sum_{j=1}^{t} \sigma^{\mathbb{Q}}(j, T) \eta_{j}+\sum_{j=1}^{t} \sigma^{\mathbb{Q}}(j, T)\left[\frac{\sum_{\tau=j}^{T} \sigma^{\mathbb{Q}}(j, \tau)+\sum_{\tau=j}^{T-1} \sigma^{\mathbb{Q}}(j, \tau)}{2}\right] .
$$

We observe that, as for the yield processes $\left\{R^{*}(\cdot, T)\right\}$, conditionally to the information $\underline{x_{0}}$, and for every maturity $T$, the processes $\{f(\cdot, T)\}$ are, under the risk-neutral probability $\mathbb{Q}$, joint MA processes driven by the same white noise $\eta_{t}$, and the past information appears through the forward rates at the date $t=0$.

If we consider $f(0, T) \equiv f_{M}(0, T)$, where $f_{M}(0, T)$ is the market forward rate at $t=0$, the process $f(\cdot, T)$ exactly fits the currently observed forward rate. With the specification of the riskneutral dynamics of the forward-rate process in terms of its volatility structure, we can represent the short-term rate process $\left(r_{t}\right)$ in the following alternative way:

Proposition 10 : Under the risk-neutral probability $\mathbb{Q}$ the short-term interest rate $r_{t+1}=f(t, t)$ is given by the expression:

$$
r_{t+1}=f(0, t)+\sum_{j=1}^{t} \sigma^{\mathbb{Q}}(j, t) \eta_{j}+\sum_{j=1}^{t} \sigma^{\mathbb{Q}}(j, t)\left[\frac{\sum_{\tau=j}^{t} \sigma^{\mathbb{Q}}(j, \tau)+\sum_{\tau=j}^{t-1} \sigma^{\mathbb{Q}}(j, \tau)}{2}\right] .
$$

One may observe that formulas (32), (33) and (34) presented above are discrete-time equivalent of classical HJM formulas [see chapter 13 in Musiela and Rutkowski (2005)] in which the conditional 
risk-neutral variance $\sigma^{\mathbb{Q}}(t+1, T)^{2}=\sigma^{2}\left(c_{1, T-t}-c_{1, T-t-1}\right)^{2}$ is a deterministic function of the time to maturity $(T-t)$ and of the parameters $\left(\sigma, \varphi^{\prime}, \gamma^{\prime}, \alpha^{\prime}\right)$.

The results presented in Sections 4.1 and 4.2 (risk-neutral MA representation of the yield and forward rate processes) can be transposed in the historical probability $(\mathbb{P})$ setting if the risk premium is constant $\left(\Gamma_{t}=\gamma_{o}\right)$ [see Appendix 5].

\section{$5 \quad$ Exact Fitting of the Initial Term Structure : Extended AR $(p)$ Approach}

In the Gaussian $\operatorname{AR}(p)$ Factor-Based Term Structure Model derived in Sections 2 and 3 the theoretical term structure may produce a poor fit of the market yield curve $R_{M}^{*}=\left\{R_{M}^{*}(0, \tau), \tau \geq 0\right\}$, while the need of an exact fitting is important in order to well price derivative securities like zero-coupon bonds and coupon bonds written on options, or caps, floor and swaptions.

We have seen in the previous section that the HJM approach leads to the exact replication of $R_{M}^{*}$ when we identify the term structure at $t=0$ with the market yield curve. Matching the theoretical and the market term structure of interest rates, at the current time $t=0$, is also possible by replacing one of the model parameters by a deterministic function of time ${ }^{6}$, and consequently, the resulting model, named Extended Gaussian AR $(p)$ Factor-Based Term Structure model, will be time-non-homogeneous. In particular, if we consider the time-homogeneous model specification (in the latent factor setting), and if we replace the parameter $\beta$, in the SDF $M_{t, t+1}$, by a timedependent function $\beta(t)$, the historical and risk-neutral dynamics of the factor $\left(x_{t}\right)$ are the same, while the short rate process, under the absence of arbitrage opportunities, is given by :

$$
r_{t+1}=\beta(t)+\alpha^{\prime} X_{t} \quad \forall t \geq 0,
$$

and it is, consequently, characterized by a non-homogeneous historical and risk-neutral dynamics. The introduction of the function $\beta(t)$ induces also the recursive equation $d_{T-t}=d_{h}$ in (10) to take a non-homogeneous specification, denoted $d(t, T)$. More precisely, following the same steps as in the proof of Proposition 1, we easily obtain that, for each maturity $T>0$, the yields to maturity associated to the extended model, are given by :

$$
R_{e}^{*}(t, T)=-\frac{c(t, T)^{\prime}}{T-t} X_{t}-\frac{d(t, T)}{T-t}
$$

where $(c(t, T), d(t, T))$ satisfy :

$$
\left\{\begin{array}{l}
c(t, T)=c_{T-t}=\Phi^{*^{\prime}} c_{T-t-1}-\alpha \\
d(t, T)=-\beta(t)+c_{1, T-t-1} \nu^{*}+\frac{1}{2} c_{1, T-t-1}^{2} \sigma^{2}+d(t+1, T),
\end{array}\right.
$$

with terminal conditions $c(T, T)=0$ and $d(T, T)=0$, for each $T>0$. Observe that the new specification of $d(t, T)$ does not create any problem for the derivation of the solution of the system $(c(t, T), d(t, T))$ : first, we solve the (time-homogeneous) difference equation for $c(t, T)=c_{h}$, as indicated in Section 3, and then we substitute backward its solution in $d(t, T)$ starting from the terminal condition $d(T, T)=0^{7}$.

\footnotetext{
${ }^{6}$ This approach is proposed in the continuous-time literature, for instance, by Ho-Lee (1986) and Dybvig (1988) [extended Merton (1970) model], Hull-White (1990, 1994) [extended Vasicek model], Hull-White (1990) and Jamshidian (1995) [extended CIR model], and by Black-Karasinski (1991) [extended log-normal Vasicek model].

${ }^{7}$ The result presented above, about the yield to maturity formula associated to the extended model, can be generalized to the case where all the model's parameters are deterministic function of time; in this case the backward solution approach is applied to both recursive relations in (37).
} 
The function $\beta(t)$, able to guarantee the exact fitting of $R_{M}^{*}$, can be chosen by means of the following two propositions.

Proposition 11 : In the univariate Extended Gaussian $\operatorname{AR}(p)$ Factor-Based Term Structure model, the yield at date $t=0$ of the zero-coupon bond maturing in $T$ can be written as :

$$
R_{e}^{*}(0, T)=\frac{1}{T} \sum_{t=0}^{T-1} \beta(t)+R_{o}^{*}(0, T), \quad \forall T>0
$$

where $R_{o}^{*}(0, T)$ is obtained from (11) with $\beta=0$, and for any value of the other parameters and $X_{0}$. [Proof : see Appendix 6].

Then, if we denote by $f_{M}=\left\{f_{M}(0, \tau), \tau \geq 0\right\}$ the market term structure of forward rates observed at date $t=0$, and by $f_{o}(0, t)=\log B_{o}^{*}(0, t)-\log B_{o}^{*}(0, t+1)$, where $B_{o}^{*}(0, t)$ is obtained from (9) with $\beta=0$, we can find the function $\beta(t)$ permitting an exact fitting.

Proposition 12 : The univariate Extended Gaussian $\operatorname{AR}(p)$ Factor-Based Term Structure model fits the currently-observed yield curve $R_{M}^{*}$ if and only if :

$$
\begin{aligned}
\beta(t) & =f_{M}(0, t)-f_{o}(0, t), \\
& =f_{M}(0, t)+\mu_{o, t}+\mu_{t}^{\prime} X_{0}, \quad \forall t \in[0, T-1],
\end{aligned}
$$

where

$$
\begin{aligned}
& \mu_{o, t}=c_{1, t} \nu^{*}+\frac{1}{2} c_{1, t}^{2} \sigma^{2} \\
& \mu_{t}=\left(\Phi^{*^{\prime}} c_{t}-c_{t}-\alpha\right)^{\prime} X_{0}
\end{aligned}
$$

[Proof : see Appendix 7].

One may observe that, by choosing $\beta(t)$ as in (39), the extended model exactly fits the observed yield curve for each possible value of $X_{0}$ and of the parameters of the model.

\section{$6 \quad$ S-Forward Dynamics}

In many financial applications, a convenient numeraire is the zero-coupon bond whose maturity $S$ is the same as the derivative to price. More precisely, the equivalent martingale measure is determined in this case, for every date $t \in[0, S]$, by the numeraire $N_{t}=\frac{B^{*}(t, S)}{B^{*}(0, S)}$, and it is referred to as $S$-forward probability and denoted by $\mathbb{Q}_{S}$. The one-period conditional (to $I_{t}$ ) density of the $S$-forward probability $\mathbb{Q}_{t, S}$ with respect to the historical probability $\mathbb{P}_{t}$, and to the risk-neutral probability $\mathbb{Q}_{t}$, are respectively given by:

$$
\begin{aligned}
\frac{d \mathbb{Q}_{t, S}}{d \mathbb{P}_{t}} & =\frac{M_{t, t+1} B^{*}(t+1, S)}{B^{*}(t, S)} \\
\frac{d \mathbb{Q}_{t, S}}{d \mathbb{Q}_{t}} & =\frac{d \mathbb{Q}_{t, S}}{d \mathbb{P}_{t}} \frac{d \mathbb{P}_{t}}{d \mathbb{Q}_{t}}=E_{t}\left(M_{t, t+1}\right) \frac{B^{*}(t+1, S)}{B^{*}(t, S)}=\exp \left(-r_{t+1}\right) \frac{B^{*}(t+1, S)}{B^{*}(t, S)} ;
\end{aligned}
$$

therefore, at the $(T-t)$-periods horizon (where $T \leq S$ ), the $S$-forward probability $\mathbb{Q}_{t, S}^{T}$ has a (conditional to $I_{t}$ ) joint density with respect to the risk-neutral probability $\mathbb{Q}_{t}^{T}$ given by:

$$
\frac{d \mathbb{Q}_{t, S}^{T}}{d \mathbb{Q}_{t}^{T}}=\prod_{\tau=t+1}^{T} \exp \left(-r_{\tau}\right) \frac{B^{*}(\tau, S)}{B^{*}(\tau-1, S)}=\frac{B^{*}(T, S)}{B^{*}(t, S)} \exp \left(-r_{t+1}-\ldots-r_{T}\right),
$$


and the pricing formula (15) takes, for $S=T$, the following useful representation:

$$
\begin{aligned}
C^{*}(t, T) & =E_{t}^{\mathbb{Q}}\left[\exp \left(-r_{t+1}-\ldots-r_{T}\right) g\left(x_{T}\right)\right] \\
& =B^{*}(t, T) E_{t}^{\mathbb{Q}_{T}}\left[g\left(x_{T}\right)\right]
\end{aligned}
$$

in which the problem of derivative pricing reduces to calculating an expectation of the payoff $g\left(x_{T}\right)$.

Proposition 13 : The $S$-forward dynamics of $x_{t+1}$ has an $\operatorname{AR}(p)$ representation of the following type:

$$
x_{t+1}=\nu_{S}+\varphi_{1}^{*} x_{t}+\ldots+\varphi_{p}^{*} x_{t+1-p}+\sigma^{*} \xi_{t+1},
$$

with

$$
\nu_{S}=\nu^{*}-\sigma^{*} \omega(t+1, S),
$$

where $\xi_{t+1} \sim \mathcal{I I N}(0,1)$ under $\mathbb{Q}_{S}\left[\right.$ Proof : see Appendix 8]. Observe that $\varepsilon_{t+1}=\xi_{t+1}-\omega(t+$ $1, S)+\Gamma_{t}$.

This model can be represented in the following vectorial form :

$$
X_{t+1}=\tilde{\nu_{S}}+\Phi^{*} X_{t}+\sigma^{*} \tilde{\xi}_{t+1},
$$

where $\tilde{\nu}_{S}=\left[\nu_{S}, 0, \ldots, 0\right]^{\prime}=\tilde{\nu}^{*}-\sigma^{*} \omega(t+1, S) e_{1}$ and $\tilde{\xi}_{t+1}=\left[\xi_{t+1}, 0, \ldots, 0\right]^{\prime}$ are $p$-dimensional vectors; $e_{1}$ denotes the first element of the canonical basis of $\mathbb{R}^{p}$.

Proposition 14 : In the $S$-forward framework, the one-period geometric zero-coupon bond return process is described by the relation:

$$
\rho(t+1, T)=-\omega(t+1, T) \xi_{t+1}+r_{t+1}-\frac{1}{2} \omega(t+1, T)^{2}+\omega(t+1, T) \omega(t+1, S),
$$

with a one-period risk premium given by :

$$
\lambda_{t}^{\mathbb{Q}_{S}}(T)=\log E_{t}^{\mathbb{Q}_{S}} \exp [\rho(t+1, T)]-r_{t+1}=\omega(t+1, T) \omega(t+1, S) .
$$

[Proof : see Appendix 9].

Consequently, under the $T$-forward probability, the one-period risk premium per unit of $\omega(t+1, T)$ is given by the $\omega(t+1, T)$ itself.

Proposition 15 : For every fixed maturity $T$, the yield process $R^{*}(\cdot, T)=\left[R^{*}(t, T), 0 \leq t \leq T\right]$, under the $S$-forward probability $\mathbb{Q}_{S}$, has the following representation:

$$
\begin{aligned}
R^{*}(t, T)=-\frac{1}{T-t} & \log \left[\frac{B^{*}(0, T)}{B^{*}(0, t)}\right]+\frac{1}{T-t} \sum_{j=1}^{t}[\omega(j, T)-\omega(j, t)] \xi_{j} \\
& \quad-\frac{1}{T-t} \sum_{j=1}^{t} \omega(j, S)[\omega(j, T)-\omega(j, t)]+\frac{1}{T-t} \frac{1}{2} \sum_{j=1}^{t}\left[\omega(j, T)^{2}-\omega(j, t)^{2}\right] .
\end{aligned}
$$

For every maturity $T$, and conditionally to the information $\underline{x_{0}}$, the processes $\left\{R^{*}(\cdot, T)\right\}$ are, also under the $S$-forward probability $\mathbb{Q}_{S}$, joint MA processes, driven by the same white noise $\xi_{t}$, and the past information is summarized in term structure at date $t=0$. [Proof : see Appendix 10.]

Corollary 3 : The zero-coupon bond price process $B^{*}(\cdot, T)=\left[B^{*}(t, T), 0 \leq t \leq T\right]$ is, for every maturity $T$ and under the $T$-forward probability $\mathbb{Q}_{T}$, given by :

$$
B^{*}(t, T)=\frac{B^{*}(0, T)}{B^{*}(0, t)} \exp \left(\sum_{j=1}^{t}[\omega(j, t)-\omega(j, T)] \xi_{j}+\frac{1}{2} \sum_{j=1}^{t}[\omega(j, t)-\omega(j, T)]^{2}\right) .
$$


Proposition 16 : For any fixed maturity $T$, the forward rate $f(t, T)=\log \left[B^{*}(t, T)\right]-\log \left[B^{*}(t, T+\right.$ $1)$ ] satisfies, under the $S$-forward probability, the following relation:

$$
\begin{gathered}
f(t, T)=f(0, T)+\sum_{j=1}^{t}[\omega(j, T+1)-\omega(j, T)] \xi_{j}+\frac{1}{2} \sum_{j=1}^{t}\left[\omega(j, T+1)^{2}-\omega(j, T)^{2}\right] \\
-\sum_{j=1}^{t} \omega(j, S)[\omega(j, T+1)-\omega(j, T)]
\end{gathered}
$$

[Proof : It follows immediately from the definition of forward rate and from Proposition 15].

Conditionally to the information $\underline{x}_{0}$, and for every maturity $T$, the processes $\{f(\cdot, T)\}$ are, also

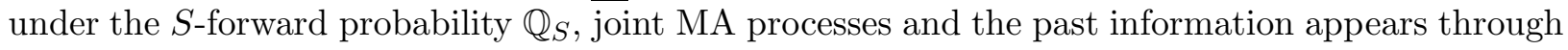
the forward rate at the date $t=0$.

If we consider $S=T+1$, relation (47) becomes :

$$
\begin{aligned}
f(t, T) & =f(0, T)+\sum_{j=1}^{t}[\omega(j, T+1)-\omega(j, T)] \xi_{j}-\frac{1}{2} \sum_{j=1}^{t}[\omega(j, T+1)-\omega(j, T)]^{2} \\
& =f(0, T)+\sum_{j=1}^{t} \sigma^{\mathbb{Q}}(j, T) \xi_{j}-\frac{1}{2} \sum_{j=1}^{t} \sigma^{\mathbb{Q}}(j, T)^{2}
\end{aligned}
$$

and, therefore, the process $\frac{B^{*}(t, T)}{B^{*}(t, T+1)}=\exp f(t, T)$ is, for every $t \leq T$, a $\mathbb{Q}_{T+1}$-martingale.

Corollary 4 : Under the $S$-forward probability $\mathbb{Q}_{S}$, the one-period forward rate increment $\Delta f(t, T)=f(t+1, T)-f(t, T)$ is given by:

$$
\Delta f(t, T)=\mu^{\mathbb{Q}_{S}}(t+1, T)+\sigma^{\mathbb{Q}_{S}}(t+1, T) \xi_{t+1},
$$

where

$$
\begin{aligned}
\sigma^{\mathbb{Q}_{S}}(t+1, T)= & \sigma^{\mathbb{Q}}(t+1, T)=\omega(t+1, T+1)-\omega(t+1, T) \\
\mu^{\mathbb{Q}_{S}}(t+1, T)= & \frac{1}{2}\left[\omega(t+1, T+1)^{2}-\omega(t+1, T)^{2}\right] \\
& -\omega(t+1, S)[\omega(t+1, T+1)-\omega(t+1, T)] \\
= & \frac{1}{2} \sigma^{\mathbb{Q}}(t+1, T)\left[\sum_{\tau=t+1}^{T} \sigma^{\mathbb{Q}}(t+1, \tau)+\sum_{\tau=t+1}^{T-1} \sigma^{\mathbb{Q}}(t+1, \tau)\right] \\
& -\sigma^{\mathbb{Q}}(t+1, T) \sum_{\tau=t+1}^{S-1} \sigma^{\mathbb{Q}}(t+1, \tau)
\end{aligned}
$$

Under the $S$-forward probability, the process $f=(f(t, T), t \geq 0)$ has independent increments and a dynamics fully specified by its conditional risk-neutral volatilities. In the particular case $S=T+1$, we have:

$$
\begin{aligned}
\sigma^{\mathbb{Q}_{T+1}}(t+1, T) & =\sigma^{\mathbb{Q}}(t+1, T), \\
\mu^{\mathbb{Q}_{T+1}}(t+1, T) & =-\frac{1}{2}[\omega(t+1, T+1)-\omega(t+1, T)]^{2}=-\frac{1}{2} \sigma^{\mathbb{Q}}(t+1, T)^{2},
\end{aligned}
$$

coherently with the martingale property of $\exp f(t, T)$ under $\mathbb{Q}_{T+1}$.

With the specification of the $(T+1)$-forward dynamics of the forward-rate process in terms of its volatility structure, we can represent the short-term rate process $\left(r_{t}\right)$ in the following way: 
Proposition 17 : Under the $(T+1)$-forward probability $\mathbb{Q}_{T+1}$, and for any maturity $T$, the short-term interest rate $r_{T+1}=f(T, T)$ is given by the expression:

$$
r_{T+1}=f(0, T)+\sum_{j=1}^{T} \sigma^{\mathbb{Q}}(j, T) \xi_{j}-\frac{1}{2} \sum_{j=1}^{T} \sigma^{\mathbb{Q}}(j, T)^{2} .
$$

We observe from (50) that $E_{0}^{\mathbb{Q}_{T+1}}\left(\exp r_{T+1}\right)=\exp f(0, T)$, that is, the linear short-term forward rate $F(0, T, T+1)$ spanning the interval $(T, T+1)$ is, under the $\mathbb{Q}_{T+1}$-forward probability, an unbiased predictor of the future linear short-term interest rate $L(T, T+1)$ spanning the same period.

\section{The Observable Factor Case}

\subsection{The Term Structure}

In what we have presented above, the factor $x_{t}$ was latent. In the term structure literature several models are specified assuming $x_{t}=r_{t+1}$ : the shape and the dynamics of the yield curve is driven by the short-rate process. This is a convenient framework, given that we can specify the historical dynamics of the factor starting from the observed stylized facts on the short-rate.

In this case, the results presented in the previous sections remain valid, except for the absence of arbitrage opportunity restriction for $r_{t+1}$, which requires $\alpha=e_{1}$ and $\beta=0$, with $e_{1}$ denoting the first element of the canonical basis of $\mathbb{R}^{p}$. Consequently, the initial conditions in the recursive equations presented in Proposition 1 become $c_{1}=-e_{1}$ and $d_{1}=0$.

In addition, the observable factor framework is useful to study how a shock on the short rate $r_{t+1}=R(t, 1)$ is propagated on the surface $R_{\mathcal{T}, \mathcal{H}}=[R(t+\tau, h), \tau \in \mathcal{T}, h \in \mathcal{H}]$, where $\mathcal{T}=$ $\{0, \ldots, T-t-1\}$ and $\mathcal{H}=(1, \ldots, H)$.

\subsection{Propagation of Short Rate Shocks on the Yield Surface}

The result presented in Proposition 1 describes, conditionally to $X_{t}$, the yields as a deterministic function of the time to maturity $h$, for a fixed date $t$. In many financial and economic contexts one needs to study which is the propagation of a shock, on the short-term interest rates, in the yield curve at different dates and for several maturities (e.g.: a Central Bank that needs to set a monetary policy). This means that we are interested in the dynamics of the process $R_{\mathcal{H}}=$ $[R(t, h), 0 \leq t<T, h \in \mathcal{H}]$, for a given set of residual times to maturity $\mathcal{H}=(1, \ldots, H)$.

If we consider a fixed time to maturity $h$, the process $R=[R(t, h), 0 \leq t<T]$ can be described by the following proposition.

Proposition 18 : For a fixed time to maturity $h$, the process $R=[R(t, h), 0 \leq t<T]$ is an $\operatorname{ARMA}(p, p-1)$ process of the following type :

$$
\Psi(L) R(t, h)=\sigma \mathbf{C}_{h}(L) \varepsilon_{t}+\mathbf{C}_{h}(1) \nu+\Psi(1) \delta_{h},
$$

where $\mathbf{C}_{h}(L)=-\left(c_{1, h}+c_{2, h} L+\ldots+c_{p, h} L^{p-1}\right) / h$ is a polynomial of degree $(p-1)$ in the lag operator $L, \delta_{h}=-\left(d_{h} / h\right)$, and where the AR polynomial, applying to $t$, is given by $\Psi(L)=$ $\left(1-\varphi_{1} L-\ldots \varphi_{p} L^{p}\right)$. [Proof: see Appendix 11.]

We observe that the AR polynomial is independent of $h$, while the MA polynomial is not. 
Proposition 19 : For a given set of residual time to maturities $\mathcal{H}=(1, \ldots, H)$, and for any status of the factor, the stochastic evolution of the yield curve process $R_{\mathcal{H}}=[R(t, h), 0 \leq t<T, h \in \mathcal{H}]$ takes the following particular $H$-variate $\operatorname{VARMA}(p, p-1)$ representation:

$$
\Psi(L)\left(\begin{array}{c}
R(t, 1) \\
R(t, 2) \\
\vdots \\
R(t, H)
\end{array}\right)=\sigma\left(\begin{array}{c}
\mathbf{C}_{1}(L) \\
\mathbf{C}_{2}(L) \\
\vdots \\
\mathbf{C}_{H}(L)
\end{array}\right) \varepsilon_{t}+\left(\begin{array}{c}
\left(\alpha^{\prime} e\right) \nu+\Psi(1) \delta_{1} \\
\mathbf{C}_{2}(1) \nu+\Psi(1) \delta_{2} \\
\vdots \\
\mathbf{C}_{H}(1) \nu+\Psi(1) \delta_{H}
\end{array}\right) .
$$

In our observable factor setting, with $x_{t}=r_{t+1}$, the yield curve process $R_{\mathcal{H}}$ is described by relation (52), with $R(t, 1)=r_{t+1}, \alpha^{\prime} e=1, \mathbf{C}_{1}(L)=1$ and $\delta_{1}=0$. Consequently, in the observable factor setting the short rate process is Markovian (of order $p$ ) by definition, while, in the latent factor framework, the short rate dynamics is non-Markovian because of the $\operatorname{ARMA}(p, p-1)$ specification.

In order to study how a shock on the short rate is propagated on the surface $R_{\mathcal{T}, \mathcal{H}}$ we need to determine, for every maturity $h \in \mathcal{H}$, the infinite moving average $[\mathrm{MA}(\infty)]$ representation of the process $R$.

Proposition 20 : Under the condition that the polynomials $\mathbf{C}_{h}(\cdot)$ and $\Psi(\cdot)$ have no common roots, and that $\Psi(z) \neq 0$ for each complex number $z$ such that $|z| \leq 1$, the process $R=[R(t, h)$, $0 \leq t<T]$ has, for each maturity $h \in \mathcal{H}$, the following $\operatorname{MA}(\infty)$ representation:

$$
R(t, h)=\Psi(1)^{-1} \mathbf{C}_{h}(1) \nu+\delta_{h}+\sigma \Theta_{h}(L) \varepsilon_{t},
$$

with

$$
\Theta_{h}(L)=\sum_{j=0}^{+\infty} \theta_{j, h} L^{j}=\Psi(L)^{-1} \mathbf{C}_{h}(L) .
$$

For each $\tau \in \mathcal{T}$ and $h \in \mathcal{H}$, the effect on $R(t+\tau, h)$ of a unit shock on $\varepsilon_{t}$ is therefore measured by the MA coefficient $\sigma \theta_{\tau, h}$.

\subsection{Exact Fitting of the Initial Term Structure in the Observable Factor Frame- work}

In Section 5 we have presented the problem of matching the initial theoretical and the market term structure of interest rates in the case where an latent factor $\left(x_{t}\right)$ drives the term structure shapes and dynamics. In particular, the fact to consider the parameter $\beta$ as the deterministic function of time derived in Proposition 12, guarantee the exact fitting of the currently-observed yield curve $R_{M}^{*}$.

In the observable factor setting, because of the absence of arbitrage opportunities $(\beta=0)$, the above mentioned approach must be applied to a different parameter. In particular, the exact fitting of $R_{M}^{*}$ is possible if we replace the parameter $\nu$ in the historical dynamics of $r_{t+1}$ by a time-dependent function $\tilde{\nu}(t)$.

Let us denote by $\left\{B_{o}^{*}(t, T), t \geq 0, T \geq t\right\}$ the term structure corresponding to any fixed values of $\left(\nu, \varphi, \sigma, \gamma_{o}, \gamma\right)$ and to the observed initial values of the short rate $\left(r_{0}, r_{-1}, \ldots, r_{-p+1}\right)$. This term structure is thus defined by the historical dynamics :

$$
\Psi(L) r_{t+1}=\nu+\sigma \varepsilon_{t}, \quad t \geq 0, \quad \varepsilon_{t+1} \sim \mathcal{I} \mathcal{I} \mathcal{N}(0,1)
$$

where $\Psi(L)=\left(1-\varphi_{1} L-\ldots \varphi_{p} L^{p}\right)$, the initial values $\left(r_{0}, r_{-1}, \ldots, r_{-p+1}\right)$, and the SDF in (5) or, equivalently, by the risk-neutral dynamics :

$$
\Psi^{*}(L) r_{t+1}=\nu+\gamma_{o} \sigma+\sigma \eta_{t}, \quad t \geq 0, \quad \eta_{t+1} \sim \mathcal{I} \mathcal{I N}(0,1)
$$


where $\Psi^{*}(L)=\left(1-\varphi_{1}^{*} L-\ldots \varphi_{p}^{*} L^{p}\right)$, with $\varphi_{i}^{*}=\varphi_{i}+\sigma \gamma_{i}$, and the initial values $\left(r_{0}, r_{-1}, \ldots, r_{-p+1}\right)$.

Let us now consider the "extended" term structure $\left\{B_{e}^{*}(t, T), t \geq 0, T \geq t\right\}$ corresponding to the time varying deterministic parameter $\nu+\tilde{\nu}(t)$, to the same parameters $\left(\varphi, \sigma, \gamma_{o}, \gamma\right)$ and the same initial values $\left(r_{0}, r_{-1}, \ldots, r_{-p+1}\right)$. This new term structure is thus defined by the historical dynamics :

$$
\Psi(L) r_{t+1} \stackrel{\mathbb{P}_{e}}{=} \nu+\tilde{\nu}(t)+\sigma \varepsilon_{t}, \quad t \geq 0, \quad \varepsilon_{t+1} \sim \mathcal{I} \mathcal{I N}(0,1)
$$

with the same initial values, and the same SDF, or, equivalently, by the risk-neutral dynamics :

$$
\Psi^{*}(L) r_{t+1} \stackrel{\mathbb{Q}_{e}}{=} \nu+\tilde{\nu}(t)+\gamma_{o} \sigma+\sigma \eta_{t}, \quad t \geq 0, \quad \eta_{t+1} \sim \mathcal{I} \mathcal{I N}(0,1)
$$

and the same initial values $\left[\mathbb{Q}_{e}\right.$ denotes the risk-neutral probability associated to the extended historical dynamics $\left.\mathbb{P}_{e}\right]$.

If we consider now the process

$$
z_{t}=r_{t}-\zeta(t-1) \text { with } t \geq-p+1,
$$

where $\zeta(t)$ is the sequence of real numbers defined by :

$$
\begin{aligned}
& \zeta(-1)=\ldots=\zeta(-p)=0 \text { and } \\
& \Psi^{*}(L) \zeta(t)=\tilde{\nu}(t), \quad t \geq 0,
\end{aligned}
$$

the risk-neutral dynamics of the process $z_{t}$ is defined by :

$$
\begin{aligned}
& \Psi^{*}(L) z_{t+1} \stackrel{\mathbb{Q}_{e}}{=} \nu+\gamma_{o} \sigma+\sigma \eta_{t}, \quad t \geq 0, \quad \eta_{t+1} \sim \mathcal{I} \mathcal{I N}(0,1) \\
& \text { with } z_{0}=r_{0}, \ldots, z_{-p+1}=r_{-p+1} .
\end{aligned}
$$

In other words, the process $\left(z_{t}\right)$ has, under $\mathbb{Q}_{e}$, exactly the same dynamics as $\left(r_{t}\right)$ under $\mathbb{Q}$, and therefore :

$$
\begin{aligned}
B_{e}^{*}(0, T) & =E_{0}^{\mathbb{Q}_{e}} \exp \left(-r_{1}-\ldots-r_{T}\right) \\
& =E_{0}^{\mathbb{Q}_{e}} \exp \left(-\sum_{t=0}^{T-1} \zeta(t)-\sum_{t=1}^{T} z_{t}\right) \\
& =\exp \left(-\sum_{t=0}^{T-1} \zeta(t)\right) E_{0}^{\mathbb{Q}} \exp \left(-\sum_{t=1}^{T} r_{t}\right) \\
& =\exp \left(-\sum_{t=0}^{T-1} \zeta(t)\right) B_{o}^{*}(0, T) .
\end{aligned}
$$

So, using the same method as in Proposition 12, we can adjust $B_{e}^{*}(0, T)$ to the market initial term structure $B_{M}^{*}(0, T)$, by choosing

$$
\zeta(t)=f_{M}(0, t)-f_{o}(0, t), \text { with } t \geq 0
$$

where $f_{M}(0, t)$ is the observed forward rate and $f_{o}(0, t)$ the forward rate associated with $B_{o}^{*}(0, t)$ [i.e., $f_{o}(0, t)=\log B_{o}^{*}(0, t)-\log B_{o}^{*}(0, t+1)$ ], or, equivalently :

$$
\begin{aligned}
& \tilde{\nu}(t)=\Psi^{*}(L) \zeta(t), \quad t \geq 0, \\
& \text { with } \zeta(t)=f_{M}(0, t)-f_{o}(0, t), \quad t \geq 0, \\
& \text { and } \zeta(-1)=\ldots=\zeta(-p)=0 .
\end{aligned}
$$


Note that $f_{o}(0, t)=r_{1}=f_{M}(0, t)$ and, therefore, $\zeta(0)=\tilde{\nu}(0)=0$.

The result is summarized in the following proposition.

Proposition 21 : For any values of the parameters $\left(\nu, \varphi, \sigma, \gamma_{o}, \gamma\right)$, the extended term structure associated with the SDF (5) and the historical dynamics :

$$
\Psi(L) r_{t+1} \stackrel{\mathbb{P}_{e}}{=} \nu+\tilde{\nu}(t)+\sigma \varepsilon_{t}, \quad t \geq 0, \quad \varepsilon_{t+1} \sim \mathcal{I} \mathcal{I} \mathcal{N}(0,1)
$$

where

$$
\begin{aligned}
& \tilde{\nu}(t)=\Psi^{*}(L) \zeta(t), \quad t \geq 0, \\
& \zeta(t)=f_{M}(0, t)-f_{o}(0, t), \quad t \geq 0, \\
& \zeta(-1)=\ldots=\zeta(-p)=0,
\end{aligned}
$$

fits exactly the observed term structure at $t=0$, if $f_{M}(0, t)$ is the observed forward rate and $f_{o}(0, t)$ the theoretical forward rate obtained from the "non extended" dynamics in which $\tilde{\nu}(t)$ is replaced by 0 , and we have $\tilde{\nu}(0)=0$.

Example : If we consider the Extended Gaussian AR(1) Factor-Based Term Structure Model, we have the discrete time equivalent of the (continuous-time) Extended Vasicek model.

Under the risk-neutral measure $\mathbb{Q}$, the dynamics of the istantaneous rate is given, for the Vasicek model, by :

$$
d r_{t}=a\left(b-r_{t}\right) d t-\sigma d W_{t}, \quad r_{0}=r>0,
$$

where $a, \sigma$ and $b$ are positive scalar coefficients, and where $W_{t}$ is a standard Brownian motion under $\mathbb{Q}$; it is well known that the risk-neutral extended dynamics is :

$$
d r_{t}=\left[\partial_{t} f_{M}(0, t)+a f_{M}(0, t)+\frac{\sigma^{2}}{2 a}(1-\exp (-2 a t))\right]-a r_{t} d t-\sigma d W_{t}
$$

where $\partial_{t} f_{M}(0, t)$ denotes the derivative of the market forward rate observed at $t=0$ [see Chapter 3 in Brigo and Mercurio (2006)].

With regard to the observable factor Extended Gaussian AR(1) Factor-Based Term Structure Model, the one-period increment is given, under $\mathbb{Q}$, by :

$$
\Delta r_{t+1}=\nu^{*}+\tilde{\nu}(t)+\left(\varphi^{*}-1\right) r_{t}+\sigma \eta_{t}, \quad \eta_{t+1} \sim \mathcal{I} \mathcal{I N}(0,1)
$$

and we know, from Proposition 21, that :

$$
\begin{aligned}
\tilde{\nu}(t) & =\left(1-\varphi^{*} L\right)\left[f_{M}(0, t)-f_{o}(0, t)\right] \\
& =f_{M}(0, t)-f_{M}(0, t-1)+\left(1-\varphi^{*}\right) f_{M}(0, t-1)-\left[f_{o}(0, t)-\varphi^{*} f_{o}(0, t-1)\right],
\end{aligned}
$$


with :

$$
\begin{aligned}
f_{o}(0, t)-\varphi^{*} f_{o}(0, t-1) & =\left[\left(c_{t}-c_{t+1}\right)-\varphi^{*}\left(c_{t-1}-c_{t}\right)\right] r_{1}+\left(d_{t}-d_{t+1}\right)-\varphi^{*}\left(d_{t-1}-d_{t}\right) \\
& =\left(d_{t}-d_{t+1}\right)-\varphi^{*}\left(d_{t-1}-d_{t}\right) \\
& =\nu^{*}\left[\varphi^{*} c_{1, t}-c_{1, t-1}\right]-\frac{\sigma^{2}}{2}\left[c_{t}^{2}-\varphi^{*} c_{t-1}^{2}\right] \\
& =\nu^{*}-\frac{\sigma^{2}}{2}\left[c_{t}^{2}-\varphi^{*} c_{t-1}^{2}\right] \\
& =\nu^{*}-\frac{\sigma^{2}}{2}\left[\frac{\left(1-\varphi^{* t}\right)^{2}}{\left(1-\varphi^{*}\right)^{2}}-\varphi^{*} \frac{\left(1-\varphi^{*(t-1)}\right)^{2}}{\left(1-\varphi^{*}\right)^{2}}\right] \\
& =\nu^{*}-\frac{\sigma^{2}}{2\left(1-\varphi^{*}\right)}\left[1-\varphi^{* 2 t-1}\right]
\end{aligned}
$$

If we substitute (69) in (67) we find the discrete-time equivalent of the extended Vasicek risk-neutral dynamics (66) :

$$
\Delta r_{t+1}=\left[\Delta f_{M}(0, t)+\left(1-\varphi^{*}\right) f_{M}(0, t-1)+\frac{\sigma^{2}}{2\left(1-\varphi^{*}\right)}\left(1-\varphi^{* 2 t-1}\right)\right]-\left(1-\varphi^{*}\right) r_{t}+\sigma \eta_{t},
$$

which is similar to $(66)$.

\section{Gaussian $\operatorname{VAR}(p)$ Factor-Based Term Structure Models}

\subsection{Historical Dynamics, SDF and Affine Term Structure}

Let us assume that the latent factor $x_{t+1}=\left(x_{1, t+1}, \ldots, x_{n, t+1}\right)^{\prime}$ driving the term structure is an $n$-dimensional $\operatorname{VAR}(p)$ process of the following type:

$$
\begin{aligned}
x_{t+1} & =\nu+\varphi_{1} x_{t}+\ldots+\varphi_{p} x_{t+1-p}+\sigma \varepsilon_{t+1} \\
& =\nu+\varphi X_{t}+\sigma \varepsilon_{t+1}
\end{aligned}
$$

where $\varepsilon_{t+1}$ is an $n$-dimensional gaussian white noise with $\mathcal{N}(0, I)$ distribution $[I$ denotes the $(n \times n)$ identity matrix]; $\sigma$ and $\varphi_{j}$, for each $j \in\{1, \ldots, p\}$, are $(n \times n)$ matrices $[\sigma$ can be chosen, for instance, lower triangular], and $\varphi=\left[\varphi_{1}, \ldots, \varphi_{p}\right]$ is an $(n \times n p)$ matrix; $\nu$ is an $n$-dimensional vector, while $X_{t}=\left(x_{t}^{\prime}, \ldots, x_{t+1-p}^{\prime}\right)^{\prime}$ is an $(n p)$-dimensional vector. This model can equivalently be represented in the following $(n p)$-dimensional $\mathrm{AR}(1)$ form :

$$
X_{t+1}=\Phi X_{t}+\left[\nu+\sigma \varepsilon_{t+1}\right] e_{1},
$$

where $e_{1}$ is a vector of size $n p$, with all entries equal to zero except for the first $n$ elements which 
are all equal to one, and where

$$
\Phi=\left[\begin{array}{cccc}
\varphi_{1} & \varphi_{2} & \ldots & \varphi_{p} \\
I_{n \times n} & \mathbf{0}_{n \times n} & \ldots & \mathbf{0}_{n \times n} \\
\mathbf{0}_{n \times n} & I_{n \times n} & \ldots & \mathbf{0}_{n \times n} \\
\vdots & \vdots & \ddots & \vdots \\
\mathbf{0}_{n \times n} & \cdots & I_{n \times n} & \mathbf{0}_{n \times n}
\end{array}\right]
$$

is a $(n p \times n p)$ matrix, with $I_{n \times n}$ the $(n \times n)$ identity matrix and $\mathbf{0}_{n \times n}$ the $(n \times n)$ matrix of zeros. Using the notation :

$$
\Gamma_{t}=\left[\Gamma_{1, t}, \ldots, \Gamma_{n, t}\right]^{\prime}
$$

where $\Gamma_{i, t}=\gamma_{o, i}+\tilde{\gamma}_{i}^{\prime} X_{t}, i \in\{1, \ldots, n\}$, and $\Gamma_{t}=\gamma_{o}+\gamma X_{t}$, with $\gamma_{o}=\left[\gamma_{o, 1}, \ldots, \gamma_{o, n}\right]^{\prime}$ an $n$ dimensional vector and $\gamma=\left[\tilde{\gamma}_{1}, \ldots, \tilde{\gamma}_{n}\right]^{\prime}$ an $(n \times n p)$ matrix, the SDF is defined as :

$$
M_{t, t+1}=\exp \left[-\beta-\alpha^{\prime} X_{t}+\Gamma_{t}^{\prime} \varepsilon_{t+1}-\frac{1}{2} \Gamma_{t}^{\prime} \Gamma_{t}\right] .
$$

Moreover, assuming the absence of arbitrage opportunities for $r_{t+1}$ we get $r_{t+1}=\beta+\alpha^{\prime} X_{t}$. It is also easy to verify that the risk premium, for an asset providing the payoff $\exp \left(-b^{\prime} x_{t+1}\right)$ at $t+1$, is $\lambda_{t}=b^{\prime} \sigma \Gamma_{t}$.

The term structure of zero coupon-bond prices is given by the following proposition :

Proposition 22 : In the Gaussian VAR $(p)$ Factor-Based Term Structure Model, the price at date $t$ of the zero-coupon bond with time to maturity $h$ is :

$$
B(t, h)=\exp \left(c_{h}^{\prime} X_{t}+d_{h}\right)
$$

where $c_{h}$ and $d_{h}$ satisfies, for $h \geq 1$, the recursive equations :

$$
\left\{\begin{aligned}
c_{h} & =-\alpha+\Phi^{\prime} c_{h-1}+(\sigma \gamma)^{\prime} c_{1, h-1} \\
& =-\alpha+\Phi^{*^{\prime}} c_{h-1}, \\
d_{h} & =-\beta+c_{1, h-1}^{\prime}\left(\nu+\sigma \gamma_{o}\right)+\frac{1}{2} c_{1, h-1}^{\prime} \sigma \sigma^{\prime} c_{1, h-1}+d_{h-1},
\end{aligned}\right.
$$

with, using the notation $\gamma=\left[\gamma_{1}, \ldots, \gamma_{p}\right]$ and where the $\gamma_{i}$ 's are $(n \times n)$ matrices :

$$
\Phi^{*}=\left[\begin{array}{cccc}
\varphi_{1}+\sigma \gamma_{1} & \varphi_{2}+\sigma \gamma_{2} & \ldots & \varphi_{p}+\sigma \gamma_{p} \\
I_{n \times n} & \mathbf{0}_{n \times n} & \ldots & \mathbf{0}_{n \times n} \\
\mathbf{0}_{n \times n} & I_{n \times n} & \ldots & \mathbf{0}_{n \times n} \\
\vdots & \vdots & \ddots & \vdots \\
\mathbf{0}_{n \times n} & \cdots & I_{n \times n} & \mathbf{0}_{n \times n}
\end{array}\right] \quad \text { a }(n p \times n p) \text { matrix }
$$

with initial conditions $c_{0}=0, d_{0}=0$ (or $c_{1}=-\alpha, d_{1}=-\beta$ ); $c_{1, h}$ indicates the vector of the first $n$ components of the $(n p)$-dimensional vector $c_{h}$. [Proof : see Appendix 13.] 
Corollary $\mathbf{5}$ : The yields to maturity associated to formula (74) are :

$$
\begin{aligned}
R(t, h) & =-\frac{1}{h} \log B(t, h) \\
& =-\frac{c_{h}^{\prime}}{h} X_{t}-\frac{d_{h}}{h}, \quad h \text { varying, }
\end{aligned}
$$

and they are affine functions of the factor $X_{t}$, that is of the $p$ most recent lagged values of the $n$-dimensional factor $x_{t+1}$.

With regard to the one-period geometric zero-coupon bond return process $\rho=[\rho(t, T), 0 \leq$ $t \leq T]$, with $\rho(t+1, T)=\log \left[B^{*}(t+1, T)\right]-\log \left[B^{*}(t, T)\right]$, it is easy to verify that :

$$
\rho(t+1, T)=r_{t+1}-\frac{1}{2} \omega(t+1, T)^{\prime} \omega(t+1, T)+\omega(t+1, T)^{\prime} \Gamma_{t}-\omega(t+1, T)^{\prime} \varepsilon_{t+1},
$$

where $\omega(t+1, T)=-\left(\sigma^{\prime} c_{1, T-t-1}\right)$ is an $n$-dimensional row vector [the proof is a generalization, to the multivariate setting, of the proof in Appendix 2]. The associated risk premium, between $t$ and $t+1$, is given by :

$$
\lambda_{t}(T)=\omega(t+1, T)^{\prime} \Gamma_{t}=\sum_{i=1}^{n} \omega_{i}(t+1, T) \Gamma_{i, t},
$$

where $\omega(t+1, T)=\left[\omega_{1}(t+1, T), \ldots, \omega_{n}(t+1, T)\right]^{\prime}$. One may notice that, in this multivariate setting, the magnitude of $\lambda_{t}(T)$ is given by a linear combination of the $n$ scalar risk premia $\Gamma_{i, t}=$ $\gamma_{o, i}+\tilde{\gamma}_{i}^{\prime} X_{t}$; moreover, for a given matrix $\gamma$ different from zero, $\lambda_{t}(T)$ is function of the $p$ most recent lagged values of the $n$-dimensional factor $x_{t+1}$.

\subsection{Risk-Neutral Dynamics}

The Laplace transform of the one-period (conditional to $\underline{x}_{t}$ ) risk-neutral distribution of $x_{t+1}$ is given by :

$$
\begin{aligned}
E_{t}^{\mathbb{Q}}\left[\exp \left(u^{\prime} x_{t+1}\right)\right] & =E_{t}\left[\frac{M_{t, t+1}}{E_{t}\left(M_{t, t+1}\right)} \exp \left(u^{\prime} x_{t+1}\right)\right] \\
& =E_{t}\left[\exp \left(\Gamma_{t}^{\prime} \varepsilon_{t+1}-\frac{1}{2} \Gamma_{t}^{\prime} \Gamma_{t}+u^{\prime} x_{t+1}\right)\right] \\
& \left.=\exp \left[u^{\prime}\left(\nu+\varphi X_{t}\right)-\frac{1}{2} \Gamma_{t}^{\prime} \Gamma_{t}\right)\right] E_{t}\left[\exp \left(\Gamma_{t}+\sigma^{\prime} u\right)^{\prime} \varepsilon_{t+1}\right] \\
& =\exp \left[u^{\prime}\left[\left(\nu+\sigma \gamma_{o}\right)+(\varphi+\sigma \gamma) X_{t}\right]+\frac{1}{2} u^{\prime} \sigma \sigma^{\prime} u\right]
\end{aligned}
$$

Therefore we get :

Proposition 23 : Under the risk neutral probability $\mathbb{Q}, x_{t+1}$ is an $n$-dimensional $\operatorname{VAR}(p)$ process of the following type:

$$
\begin{aligned}
x_{t+1} & =\nu^{*}+\varphi_{1}^{*} x_{t}+\ldots+\varphi_{p}^{*} x_{t+1-p}+\sigma^{*} \eta_{t+1} \\
& =\nu^{*}+\varphi^{*} X_{t}+\sigma^{*} \eta_{t+1},
\end{aligned}
$$


with

$$
\begin{aligned}
\nu^{*} & =\left(\nu+\sigma \gamma_{o}\right) \\
\varphi_{j}^{*} & =\left(\varphi_{j}+\sigma \gamma_{j}\right), \quad \text { for } j \in\{1, \ldots, p\} \\
\varphi^{*} & =\left[\varphi_{1}^{*}, \ldots, \varphi_{p}^{*}\right] \\
\sigma^{*} & =\sigma,
\end{aligned}
$$

where $\eta_{t+1}$ is (under $\mathbb{Q}$ ) an $n$-dimensional gaussian white noise with $\mathcal{N}(0, I)$ distribution, and $I$ denotes the $(n \times n)$ identity matrix.

This model can be represented in the following vectorial form :

$$
X_{t+1}=\Phi^{*} X_{t}+\left[\nu^{*}+\sigma^{*} \eta_{t+1}\right] e_{1}
$$

where $e_{1}$ is the vector of size $n p$, with all entries equal to zero except for the first $n$ elements which are all equal to one, and where $\Phi^{*}$ is defined in Proposition 22.

With regard to the one-period zero-coupon bond return process, under the risk-neutral probability we have that :

$$
\rho(t+1, T)=r_{t+1}-\frac{1}{2} \omega(t+1, T)^{\prime} \omega(t+1, T)-\omega(t+1, T)^{\prime} \eta_{t+1},
$$

with a risk premium $\lambda_{t}^{\mathbb{Q}}(T)=0$ [the proof is a generalization, to the multivariate setting, of the proof in Appendix 3].

\subsection{Moving Average or HJM Representations in the Multivariate Framework}

The purpose of this section is to generalize the results presented in Section 4 to the case where the factor $\left(x_{t}\right)$ is the $n$-dimensional process defined in (71). Following the same steps as in the univariate setting, and using relation (81), we have :

Proposition 24 : In the Gaussian $\operatorname{VAR}(p)$ Factor-Based Term Structure Model, for every fixed maturity $T$ and under the risk-neutral probability $\mathbb{Q}$ :

- the yield process $R^{*}(\cdot, T)=\left[R^{*}(t, T), 0 \leq t \leq T\right]$ has the following representation:

$$
\begin{array}{r}
R^{*}(t, T)=-\frac{1}{T-t} \log \left[\frac{B^{*}(0, T)}{B^{*}(0, t)}\right]+\frac{1}{T-t} \sum_{j=1}^{t}[\omega(j, T)-\omega(j, t)]^{\prime} \eta_{j} \\
+\frac{1}{T-t} \frac{1}{2} \sum_{j=1}^{t}\left[\omega(j, T)^{\prime} \omega(j, T)-\omega(j, t)^{\prime} \omega(j, t)\right],
\end{array}
$$

where $\eta_{j} \stackrel{\mathbb{Q}}{\sim} \mathcal{I} \mathcal{I} \mathcal{N}(0, I)$. Conditionally to the information $x_{0}$, and for every maturity $T$, the processes $\left\{R^{*}(\cdot, T)\right\}$ are, under the risk-neutral probability $\mathbb{Q}$, joint MA processes, driven by a $n$-dimensional gaussian white noise, with time-varying coefficients, and the past information appears through the term structure at date $t=0$; 
- the forward rate process $f(t, T)=\log \left[B^{*}(t, T)\right]-\log \left[B^{*}(t, T+1)\right]$ satisfies :

$$
\begin{aligned}
f(t, T)=f(0, T)+\sum_{j=1}^{t}[\omega(j, T+1)-\omega(j, T)]^{\prime} \eta_{j} \\
\quad+\frac{1}{2} \sum_{j=1}^{t}\left[\omega(j, T+1)^{\prime} \omega(j, T+1)-\omega(j, T)^{\prime} \omega(j, T)\right] \\
=f(0, T)+\sum_{j=1}^{t} \sigma^{\mathbb{Q}}(j, T)^{\prime} \eta_{j} \\
\quad+\frac{1}{2} \sum_{j=1}^{t} \sigma^{\mathbb{Q}}(j, T)^{\prime}\left[\sum_{\tau=j}^{T} \sigma^{\mathbb{Q}}(j, \tau)+\sum_{\tau=j}^{T-1} \sigma^{\mathbb{Q}}(j, \tau)\right]
\end{aligned}
$$

where $f(0, T)=\log \left[\frac{B^{*}(0, T)}{B^{*}(0, T+1)}\right]$ and $\sigma^{\mathbb{Q}}(j, \tau)=\omega(j, \tau+1)-\omega(j, \tau)$ for each $j \leq \tau \leq T$.

As for the yield processes $\left\{R^{*}(\cdot, T)\right\}$, conditionally to the information $\underline{x_{0}}$, and for every maturity $T$, the processes $\{f(\cdot, T)\}$ are, under the risk-neutral probability $\overline{\mathbb{Q}}$, joint MA processes and the past information appears through the forward rates at the date $t=0$.

- the one-period forward rate increment $\Delta f(t, T)=f(t+1, T)-f(t, T)$ is give by:

$$
\Delta f(t, T)=\mu^{\mathbb{Q}}(t+1, T)+\sigma^{\mathbb{Q}}(t+1, T)^{\prime} \eta_{t+1},
$$

where

$$
\begin{aligned}
\sigma^{\mathbb{Q}}(t+1, T) & =\omega(t+1, T+1)-\omega(t+1, T) \\
\mu^{\mathbb{Q}}(t+1, T) & =\frac{1}{2}\left[\omega(t+1, T+1)^{\prime} \omega(t+1, T+1)-\omega(t+1, T)^{\prime} \omega(t+1, T)\right] \\
& =\frac{1}{2} \sigma^{\mathbb{Q}}(t+1, T)^{\prime} \times\left[\sum_{\tau=t+1}^{T} \sigma^{\mathbb{Q}}(t+1, \tau)+\sum_{\tau=t+1}^{T-1} \sigma^{\mathbb{Q}}(t+1, \tau)\right]
\end{aligned}
$$

- the short-term interest rate $r_{t+1}=f(t, t)$ is given by the expression:

$$
r_{t+1}=f(0, t)+\sum_{j=1}^{t} \sigma^{\mathbb{Q}}(j, t)^{\prime} \eta_{j}+\frac{1}{2} \sum_{j=1}^{t} \sigma^{\mathbb{Q}}(j, t)^{\prime}\left[\sum_{\tau=j}^{t} \sigma^{\mathbb{Q}}(j, \tau)+\sum_{\tau=j}^{t-1} \sigma^{\mathbb{Q}}(j, \tau)\right] .
$$

\subsection{S-Forward Dynamics}

The $S$-forward dynamics of the $n$-dimensional factor $x_{t+1}$, has an $\operatorname{VAR}(p)$ representation of the following type:

$$
x_{t+1}=\nu_{S}+\varphi_{1}^{*} x_{t}+\ldots+\varphi_{p}^{*} x_{t+1-p}+\sigma^{*} \xi_{t+1}
$$

with

$$
\nu_{S}=\nu^{*}-\sigma^{*} \omega(t+1, S),
$$

where $\xi_{t+1} \sim \mathcal{I} \mathcal{I N}(0, I)$ under $\mathbb{Q}_{S}$ [the proof is a generalization, to the multivariate setting, of the proof in Appendix 8].

This model can be represented in the following vectorial form :

$$
X_{t+1}=\Phi^{*} X_{t}+\left[\nu_{S}+\sigma^{*} \xi_{t+1}\right] e_{1},
$$


where $e_{1}$ denotes the vector of size $n p$, with all entries equal to zero except for the first $n$ elements which are all equal to one.

Moreover, the one-period geometric zero-coupon bond return process is given by:

$$
\begin{gathered}
\rho(t+1, T)=r_{t+1}-\omega(t+1, T)^{\prime} \xi_{t+1}-\frac{1}{2} \omega(t+1, T)^{\prime} \omega(t+1, T) \\
+\omega(t+1, T)^{\prime} \omega(t+1, S),
\end{gathered}
$$

with one-period risk premium given by :

$$
\lambda_{t}^{\mathbb{Q}_{S}}(T)=\log E_{t}^{\mathbb{Q}_{S}} \exp [\rho(t+1, T)]-r_{t+1}=\omega(t+1, T)^{\prime} \omega(t+1, S) .
$$

Starting from relations (87) and (88), we can determine the generalizations, to the multivariate $S$-forward framework, of the results presented in Section 6. More precisely, we have :

Proposition 25 : In the Gaussian $\operatorname{VAR}(p)$ Factor-Based Term Structure Model, for every fixed maturity $T$ and under the $S$-forward probability $\mathbb{Q}_{S}$ :

- the yield process $R^{*}(\cdot, T)=\left[R^{*}(t, T), 0 \leq t \leq T\right]$ has the following representation:

$$
\begin{aligned}
R^{*}(t, T)=-\frac{1}{T-t} & \log \left[\frac{B^{*}(0, T)}{B^{*}(0, t)}\right]+\frac{1}{T-t} \sum_{j=1}^{t}[\omega(j, T)-\omega(j, t)]^{\prime} \xi_{j} \\
& -\frac{1}{T-t} \sum_{j=1}^{t}[\omega(j, T)-\omega(j, t)]^{\prime} \omega(j, S) \\
& +\frac{1}{T-t} \frac{1}{2} \sum_{j=1}^{t}\left[\omega(j, T)^{\prime} \omega(j, T)-\omega(j, t)^{\prime} \omega(j, t)\right] ;
\end{aligned}
$$

for every maturity $T$, and conditionally to the information $\underline{x_{0}}$, the processes $\left\{R^{*}(\cdot, T)\right\}$ are, also under the $S$-forward probability $\mathbb{Q}_{S}$, joint MA processes, driven by a $n$-dimensional gaussian white noise, and the past information is summarized in term structure at date $t=0$

- the forward rate $f(t, T)=\log \left[B^{*}(t, T)\right]-\log \left[B^{*}(t, T+1)\right]$ satisfies the following relation:

$$
\begin{aligned}
f(t, T)=f(0, & T)+\sum_{j=1}^{t}[\omega(j, T+1)-\omega(j, T)]^{\prime} \xi_{j} \\
& +\frac{1}{2} \sum_{j=1}^{t}\left[\omega(j, T+1)^{\prime} \omega(j, T+1)-\omega(j, T)^{\prime} \omega(j, T)\right] \\
& -\sum_{j=1}^{t}[\omega(j, T+1)-\omega(j, T)]^{\prime} \omega(j, S) ;
\end{aligned}
$$

Conditionally to the information $\underline{x_{0}}$, and for every maturity $T$, the processes $\{f(\cdot, T)\}$ are, also under the $S$-forward probability $\mathbb{Q}_{S}$, joint MA processes and the past information appears through the forward rate at the date $t=0$.

If we consider $S=T+1$, relation (90) becomes:

$$
\begin{aligned}
f(t, T)= & f(0, T)+\sum_{j=1}^{t}[\omega(j, T+1)-\omega(j, T)]^{\prime} \xi_{j} \\
& \quad-\frac{1}{2} \sum_{j=1}^{t}[\omega(j, T+1)-\omega(j, T)]^{\prime}[\omega(j, T+1)-\omega(j, T)] \\
= & f(0, T)+\sum_{j=1}^{t} \sigma^{\mathbb{Q}}(j, T)^{\prime} \xi_{j}-\frac{1}{2} \sum_{j=1}^{t} \sigma^{\mathbb{Q}}(j, T)^{\prime} \sigma^{\mathbb{Q}}(j, T),
\end{aligned}
$$

and, therefore, the process $\frac{B^{*}(t, T)}{B^{*}(t, T+1)}=\exp f(t, T)$ is, for every $t \leq T$, a $\mathbb{Q}_{T+1}$-martingale. 
- the one-period forward rate increment $\Delta f(t, T)=f(t+1, T)-f(t, T)$ is given by:

$$
\Delta f(t, T)=\mu^{\mathbb{Q}_{S}}(t+1, T)+\sigma^{\mathbb{Q}_{S}}(t+1, T)^{\prime} \xi_{t+1},
$$

where

$$
\begin{aligned}
& \sigma^{\mathbb{Q}_{S}}(t+1, T)= \sigma^{\mathbb{Q}}(t+1, T)=\omega(t+1, T+1)-\omega(t+1, T) \\
& \mu^{\mathbb{Q}_{S}}(t+1, T)= \frac{1}{2}\left[\omega(t+1, T+1)^{\prime} \omega(t+1, T+1)-\omega(t+1, T)^{\prime} \omega(t+1, T)\right] \\
& \quad-[\omega(t+1, T+1)-\omega(t+1, T)]^{\prime} \omega(t+1, S) \\
&=\frac{1}{2} \sigma^{\mathbb{Q}}(t+1, T)^{\prime} \times\left[\sum_{\tau=t+1}^{T} \sigma^{\mathbb{Q}}(t+1, \tau)+\sum_{\tau=t+1}^{T-1} \sigma^{\mathbb{Q}}(t+1, \tau)\right] \\
& \quad-\sigma^{\mathbb{Q}}(t+1, T)^{\prime} \sum_{\tau=t+1}^{S-1} \sigma^{\mathbb{Q}}(t+1, \tau),
\end{aligned}
$$

the process $f=(f(t, T), t \geq 0)$ has independent increments and a dynamics fully specified by its conditional risk-neutral volatilities. In the particular case $S=T+1$, we have:

$$
\begin{aligned}
\sigma^{\mathbb{Q}_{T+1}}(t+1, T) & =\sigma^{\mathbb{Q}}(t+1, T), \\
\mu^{\mathbb{Q}_{T+1}}(t+1, T) & =-\frac{1}{2} \sigma^{\mathbb{Q}}(t+1, T)^{\prime} \sigma^{\mathbb{Q}}(t+1, T),
\end{aligned}
$$

coherently with the martingale property of $\exp f(t, T)$ under $\mathbb{Q}_{T+1}$.

- for $S=T+1$, the short-term interest rate $r_{T+1}=f(T, T)$ is given by the expression:

$$
r_{T+1}=f(0, T)+\sum_{j=1}^{T} \sigma^{\mathbb{Q}}(j, T)^{\prime} \xi_{j}-\frac{1}{2} \sum_{j=1}^{T} \sigma^{\mathbb{Q}}(j, T)^{\prime} \sigma^{\mathbb{Q}}(j, T) .
$$

\subsection{The Observable Factor Case}

In the multivariate observable factor setting, the $n$-dimensional factor $\left(x_{t}\right)$ can be considered as a vector of yields at different maturities in which the first component is assumed to be the short rate $r_{t+1}$; more precisely, we assume:

$$
x_{t}=\left[\begin{array}{c}
R\left(t, h_{1}\right) \\
R\left(t, h_{2}\right) \\
\vdots \\
R\left(t, h_{n}\right)
\end{array}\right]
$$

where $R(t, 1)=r_{t+1}$ and $h_{1}<h_{2}<\ldots<h_{n}$. In this case, the absence of arbitrage conditions for the $n$ yields in (94) imply :

$$
\begin{aligned}
& \text { (i) } c_{1}=-e_{1}, \quad d_{1}=0, \\
& \text { (ii) } c_{h_{j}}=-h_{j} e_{h_{j}}, \quad d_{h_{j}}=0, \forall j \in\{2, \ldots, n\},
\end{aligned}
$$

where $e_{h_{j}}$ denotes the $h_{j}^{t h}$ element of the canonical basis in $\mathbb{R}^{n p}$. The first set of conditions is used as initial value in the recursive formula of Proposition 22; the second set of $(n-1)$ conditions imply restrictions on the parameters $\left(\Phi^{*}, \nu^{*}, \sigma^{*}\right)$ which must be taken into account at the estimation stage. 


\section{Conclusions}

The purpose of this paper was to generalize the Gaussian family of discrete-time affine term structure models by the introduction of lags in the historical and risk-neutral factor dynamics, and in the specification of the stochastic risk correction coefficients.

We have studied the Gaussian $\operatorname{AR}(p)$ and $\operatorname{VAR}(p)$ Factor-Based Term Structure Models, and we have verified the important role played by the autoregressive order in the replication of term structure shapes coherent with observations. Moreover, several characterizations of the yield and short-term forward rate processes, under the risk-neutral (Moving Average representation) and $S$ forward probability, are proposed. We have also studied the problem of exact fitting of the initial term structure by means of the Extended $\operatorname{AR}(p)$ approach. These results are given for a latent factor, and for an observable factor. In the second case the factor is the short rate (scalar setting) or a vector of several yields (multivariate framework). 


\section{Appendix 1 : Proof of Proposition 1}

Assuming that (9) is true for $h-1$, we get:

$$
\begin{aligned}
B(t, h) & =\exp \left(c_{h}^{\prime} X_{t}+d_{h}\right) \\
& =E_{t}\left[M_{t, t+1} \cdots M_{t+H-1, t+H}\right] \\
& =E_{t}\left[M_{t, t+1} B(t+1, h-1)\right] \\
& =\exp \left[-\beta-\alpha^{\prime} X_{t}-\frac{1}{2} \Gamma_{t}^{2}+d_{h-1}\right] \times E_{t}\left[\exp \left(\Gamma_{t} \varepsilon_{t+1}+c_{h-1}^{\prime} X_{t+1}\right)\right] \\
& \left.=\exp \left[-\beta-\alpha^{\prime} X_{t}-\frac{1}{2} \Gamma_{t}^{2}+d_{h-1}+c_{h-1}^{\prime}\left(\Phi X_{t}+\tilde{\nu}\right)\right] \times E_{t}\left[\exp \left(\Gamma_{t}+\sigma c_{1, h-1}\right) \varepsilon_{t+1}\right)\right] \\
& =\exp \left[\left(-\alpha+\Phi^{\prime} c_{h-1}+c_{1, h-1} \sigma \gamma\right)^{\prime} X_{t}\right. \\
& \left.\quad+\left(-\beta+c_{1, h-1} \nu+\frac{1}{2} c_{1, h-1}^{2} \sigma^{2}+\gamma_{o} c_{1, h-1} \sigma+d_{h-1}\right)\right]
\end{aligned}
$$

and by identifying the coefficients we find the recursive relation presented in Proposition 1.

\section{Appendix 2 : Proof of Proposition 2}

$$
\begin{aligned}
\rho(t+1, T) & =\log \left[B^{*}(t+1, T)\right]-\log \left[B^{*}(t, T)\right] \\
& =c_{T-t-1}^{\prime} X_{t+1}+d_{T-t-1}-c_{T-t}^{\prime} X_{t}-d_{T-t} \\
& =c_{T-t-1}^{\prime}\left[X_{t+1}-\Phi X_{t}-\tilde{\nu}\right]+\left(\beta+\alpha^{\prime} X_{t}\right)-\sigma c_{1, T-t-1}\left(\gamma_{o}+\gamma^{\prime} X_{t}\right)-\frac{1}{2} c_{1, T-t-1}^{2} \sigma^{2} \\
& =\left(c_{1, T-t-1} \sigma\right) \varepsilon_{t+1}+\left(\beta+\alpha^{\prime} X_{t}\right)-\sigma c_{1, T-t-1}\left(\gamma_{o}+\gamma^{\prime} X_{t}\right)-\frac{1}{2} c_{1, T-t-1}^{2} \sigma^{2} .
\end{aligned}
$$

Now, we have that, under the absence of arbitrage $r_{t+1}=\left(\beta+\alpha^{\prime} X_{t}\right)$ and, consequently, the Proposition 2 is proved.

\section{Appendix 3 : Proof of Proposition 4}

$$
\begin{aligned}
\rho(t+1, T) & =\log \left[B^{*}(t+1, T)\right]-\log \left[B^{*}(t, T)\right] \\
& =c_{T-t-1}^{\prime} X_{t+1}+d_{T-t-1}-c_{T-t}^{\prime} X_{t}-d_{T-t} \\
& =c_{T-t-1}^{\prime} X_{t+1}-\left(-\alpha^{\prime}+c_{T-t-1}^{\prime} \Phi^{*}\right) X_{t}+\beta-c_{1, T-t-1} \nu^{*}-\frac{1}{2} c_{1, T-t-1}^{2} \sigma^{2} \\
& =c_{T-t-1}^{\prime}\left[X_{t+1}-\Phi^{*} X_{t}-\tilde{\nu}^{*}\right]+r_{t+1}-\frac{1}{2} c_{1, T-t-1}^{2} \sigma^{2} \\
& =r_{t+1}-\frac{1}{2} \omega(t+1, T)^{2}-\omega(t+1, T) \eta_{t+1} .
\end{aligned}
$$




\section{Appendix 4 : Proof of Proposition 9}

The conditional risk-neutral mean of the one-period forward rate increment

$$
\Delta f(t, T)=\mu^{\mathbb{Q}}(t+1, T)+\sigma^{\mathbb{Q}}(t+1, T) \eta_{t+1}
$$

can be written in the following way:

$$
\begin{aligned}
\mu^{\mathbb{Q}}(t+1, T) & =\frac{1}{2}\left[\omega(t+1, T+1)^{2}-\omega(t+1, T)^{2}\right] \\
& =\frac{1}{2} \sigma^{\mathbb{Q}}(t+1, T)[\omega(t+1, T+1)+\omega(t+1, T)],
\end{aligned}
$$

and, given that $\omega(i, i)=0$, we can write

$$
\omega(t+1, T+1)=\sum_{\tau=t+1}^{T} \sigma^{\mathbb{Q}}(t+1, \tau) \quad \forall t \leq T
$$

consequently, relation (A.4) can be represented in the following way:

$$
\mu^{\mathbb{Q}}(t+1, T)=\frac{1}{2} \sigma^{\mathbb{Q}}(t+1, T)\left[\sum_{\tau=t+1}^{T} \sigma^{\mathbb{Q}}(t+1, \tau)+\sum_{\tau=t+1}^{T-1} \sigma^{\mathbb{Q}}(t+1, \tau)\right],
$$

and Proposition 9 is proved.

\section{Appendix 5 : Historical MA and HJM representations}

\section{A.5.1 MA REPRESENTATION OF THE HistoriCAL YIELD PROCESS}

In this appendix we want to determine the conditions under which the joint historical dynamics of the yield processes $R^{*}(\cdot, T)=\left[R^{*}(t, T), 0 \leq t \leq T\right]$, with $R^{*}(t, T)=-\frac{1}{T-t} \log B^{*}(t, T)$ and $R^{*}(t, T)=R(t, T-t)$, can be represented under a Moving Average form. The derivation of the joint dynamics of the processes $R^{*}(\cdot, T)$ is based on the representation of the log-price stochastic process in terms of the conditional variances of $\rho=[\rho(t, T), 0 \leq t \leq T]$, where $\rho(t+1, T)=$ $\log \left[B^{*}(t+1, T)\right]-\log \left[B^{*}(t, T)\right]$. Starting from relation (12) we can write the log-price as :

$$
\begin{aligned}
\log \left[B^{*}(t, T)\right]= & \sum_{j=1}^{t} \rho(j, T)+\log \left[B^{*}(0, T)\right] \\
= & -\sum_{j=1}^{t} \omega(j, T) \varepsilon_{j}+\sum_{j=1}^{t} r_{j}+\sum_{j=1}^{t} \omega(j, T)\left(\gamma_{o}+\gamma^{\prime} X_{j-1}\right) \\
& -\frac{1}{2} \sum_{j=1}^{t} \omega(j, T)^{2}+\log \left[B^{*}(0, T)\right],
\end{aligned}
$$

and, consequently, we can give the following proposition.

Proposition A.1 : For every fixed maturity $T$, the zero-coupon bond price process $B^{*}(\cdot, T)=$ $\left[B^{*}(t, T), 0 \leq t \leq T\right]$, under the historical probability $\mathbb{P}$, has the following specification:

$$
B^{*}(t, T)=B^{*}(0, T) \exp \left[\sum_{j=1}^{t}\left[r_{j}-\frac{1}{2} \omega(j, T)^{2}+\omega(j, T) \Gamma_{j-1}\right]-\sum_{j=1}^{t} \omega(j, T) \varepsilon_{j}\right] .
$$

If we put $T=t$ in (A.5), we find a relation for the sum of the short-rates:

$$
\sum_{j=1}^{t} r_{j}=\sum_{j=1}^{t} \omega(j, t) \varepsilon_{j}-\sum_{j=1}^{t} \omega(j, t)\left(\gamma_{o}+\gamma^{\prime} X_{j-1}\right)+\frac{1}{2} \sum_{j=1}^{t} \omega(j, t)^{2}-\log \left[B^{*}(0, t)\right]
$$


that we can substitute in (A.5) to find, for every date $t$ and maturity $T>t$ :

$$
\begin{aligned}
\log \left[B^{*}(t, T)\right]=\log [ & \left.\frac{B^{*}(0, T)}{B^{*}(0, t)}\right]-\sum_{j=1}^{t}[\omega(j, T)-\omega(j, t)] \varepsilon_{j} \\
& +\sum_{j=1}^{t}[\omega(j, T)-\omega(j, t)]\left(\gamma_{o}+\gamma^{\prime} X_{j-1}\right)-\frac{1}{2} \sum_{j=1}^{t}\left[\omega(j, T)^{2}-\omega(j, t)^{2}\right] .
\end{aligned}
$$

Conditionally to the information $\underline{x_{0}}$, and for every maturity $T$, the processes $\left\{R^{*}(\cdot, T)\right\}$ are, under the historical probability $\mathbb{P}$, joint $\bar{M}$ A processes with time-varying coefficients (driven by the same white noise $\varepsilon_{t}$ ) only if we assume $\gamma=0$.

\section{A.5.2 The HJM REPRESENTATION OF HistoriCAL SHORT-TERM FORWARD RATES}

Starting from the one-period forward rate $f(t, T)=\log \left[B^{*}(t, T)\right]-\log \left[B^{*}(t, T+1)\right]$, if we use relation (A.8) we have :

Proposition A.2 : Under the historical probability $\mathbb{P}$, and for any fixed maturity $T$, the forward rate $f(t, T)$ satisfies the following relation:

$$
\begin{aligned}
& f(t, T)=f(0, T)+\sum_{j=1}^{t}[\omega(j, T+1)-\omega(j, T)] \varepsilon_{j} \\
& \quad-\sum_{j=1}^{t}[\omega(j, T+1)-\omega(j, T)]\left(\gamma_{o}+\gamma^{\prime} X_{j-1}\right)+\frac{1}{2} \sum_{j=1}^{t}\left[\omega(j, T+1)^{2}-\omega(j, T)^{2}\right],
\end{aligned}
$$

where $f(0, T)=\log \left[\frac{B^{*}(0, T)}{B^{*}(0, T+1)}\right]$.

Corollary A.1 : The one-period forward rate increment $\Delta f(t, T)=f(t+1, T)-f(t, T)$, is given by :

$$
\Delta f(t, T)=\mu^{\mathbb{P}}(t+1, T)+\sigma^{\mathbb{P}}(t+1, T) \varepsilon_{t+1},
$$

where

$$
\begin{aligned}
& \sigma^{\mathbb{P}}(t+1, T)= {[\omega(t+1, T+1)-\omega(t+1, T)]=\sigma^{\mathbb{Q}}(t+1, T) } \\
& \mu^{\mathbb{P}}(t+1, T)= \frac{1}{2}\left[\omega(t+1, T+1)^{2}-\omega(t+1, T)^{2}\right]-\sigma^{\mathbb{P}}(t+1, T) \Gamma_{t} \\
&= \mu^{\mathbb{Q}}(t+1, T)-\sigma^{\mathbb{P}}(t+1, T) \Gamma_{t} \\
&= \sigma^{\mathbb{Q}}(t+1, T)\left[\frac{\sum_{\tau=t+1}^{T} \sigma^{\mathbb{Q}}(t+1, \tau)+\sum_{\tau=t+1}^{T-1} \sigma^{\mathbb{Q}}(t+1, \tau)}{2}\right] \\
&-\sigma^{\mathbb{Q}}(t+1, T) \Gamma_{t}
\end{aligned}
$$

We observe that, as for the historical yield processes $\left\{R^{*}(\cdot, T)\right\}$, conditionally to the information $x_{0}$, and for every maturity $T$, the processes $\{f(\cdot, T)\}$ are, under the probability $\mathbb{P}$, joint MA processes (driven by the same white noise $\varepsilon_{t}$ ) only in the case $\Gamma_{t}=\gamma_{o}$. 


\section{Appendix 6 : Proof of Proposition 11}

Denoting $B_{e}^{*}(0, T)$ the zero coupon price in the extended model, we have :

$$
\begin{aligned}
& B_{e}^{*}(0, T) \\
= & E_{0}^{\mathbb{Q}}\left[\exp \left(-r_{1}-\ldots-r_{T}\right)\right] \\
= & \exp \left[-\sum_{t=0}^{T-1} \beta(t)\right] E_{0}^{\mathbb{Q}}\left[\exp \left(-\alpha^{\prime} X_{0}-\ldots-\alpha^{\prime} X_{T-1}\right)\right] \\
= & \exp \left[-\sum_{t=0}^{T-1} \beta(t)\right] E_{0}\left[\frac{M_{0,1} \cdot \ldots \cdot M_{T-1, T}}{E_{t}\left(M_{0,1}\right) \cdot \ldots \cdot E_{T-1}\left(M_{T-1, T}\right)} \exp \left(-\alpha^{\prime} X_{0}-\ldots-\alpha^{\prime} X_{T-1}\right)\right] \\
= & \exp \left[-\sum_{t=0}^{T-1} \beta(t)\right] E_{0}\left[M_{0,1}^{o} \cdot \ldots \cdot M_{T-1, T}^{o}\right] \\
= & \exp \left[-\sum_{t=0}^{T-1} \beta(t)\right] B_{o}^{*}(0, T),
\end{aligned}
$$

where $B_{o}^{*}(0, T)=E_{0}\left[M_{0,1}^{o} \cdot \ldots \cdot M_{T-1, T}^{o}\right]$ is the price at date $t=0$ of the zero-coupon bond maturing in $T$ and obtained with the pricing kernel (4) in which we assume $\beta=0$. The result in Proposition 11 follows from $R_{e}^{*}(0, T)=-\log \left(B_{e}^{*}(0, T)\right) / T$.

\section{Appendix 7 : Proof of Proposition 12}

We are searching for the values of $\beta(t)$ such that $R_{e}^{*}(0, t)=R_{M}^{*}(0, t)$ for all $t$. From the result of Proposition 11, we can equivalently rewrite the above matching condition in the following way :

$$
\begin{aligned}
f_{M}(0, t) & =f_{e}(0, t) \\
& =\log B_{e}(0, t)-\log B_{e}(0, t+1)=\beta(t)+f_{o}(0, t)
\end{aligned}
$$

and consequently, for each date $t$, we can always choose the matching function $\beta(t)=f_{M}(0, t)-$ $f_{o}(0, t)$. Now, from formula $(9)$, with $\beta=0$, we obtain:

$$
\begin{aligned}
\beta(t) & =f_{M}(0, t)-\left[\left(c_{t}-c_{t+1}\right)^{\prime} X_{0}+\left(d_{t}-d_{t+1}\right)\right] \\
& =f_{M}(0, t)+\left(\Phi^{*} c_{t}-c_{t}-\alpha\right)^{\prime} X_{0}+\left(c_{1, t} \nu^{*}+\frac{1}{2} c_{1, t}^{2} \sigma^{2}\right) \\
& =f_{M}(0, t)+\mu_{o, t}+\mu_{t}^{\prime} X_{0},
\end{aligned}
$$

with

$$
\begin{aligned}
& \mu_{o, t}=c_{1, t} \nu^{*}+\frac{1}{2} c_{1, t}^{2} \sigma^{2} \\
& \mu_{t}=\left(\Phi^{*^{\prime}} c_{t}-c_{t}-\alpha\right)^{\prime} X_{0}
\end{aligned}
$$

and the result in Proposition 12 is proved. 


\section{Appendix 8 : Proof of Proposition 13}

The $S$-forward Laplace transform of $x_{t+1}$, conditionally to $I_{t}=\left(\underline{x_{t}}\right)$, can be written in the following way:

$$
\begin{aligned}
& E_{t}^{\mathbb{Q}_{S}}\left[\exp \left(u x_{t+1}\right)\right] \\
= & \frac{1}{B^{*}(t, S)} E_{t}^{\mathbb{Q}}\left[\exp \left(-r_{t+1}-\ldots-r_{S}+u x_{t+1}\right)\right] .
\end{aligned}
$$

From relation $(28)$, we have that, under the risk-neutral measure $\mathbb{Q}$, the sum of short-term rates in the above formula can be written as:

$$
\begin{aligned}
\sum_{j=t+1}^{S} r_{j}= & \sum_{j=1}^{S} r_{j}-\sum_{j=1}^{t} r_{j} \\
= & \sum_{j=1}^{S} \omega(j, S) \eta_{j}-\sum_{j=1}^{t} \omega(j, t) \eta_{j} \\
& \quad+\frac{1}{2}\left[\sum_{j=1}^{S} \omega(j, S)^{2}-\sum_{j=1}^{t} \omega(j, t)^{2}\right]+\log \left[\frac{B^{*}(0, t)}{B^{*}(0, S)}\right]
\end{aligned}
$$

and, consequently, we get:

$$
\begin{aligned}
& E_{t}^{\mathbb{Q} S}\left[\exp \left(u x_{t+1}\right)\right] \\
= & \frac{\exp \left[-\frac{1}{2}\left[\sum_{j=1}^{S} \omega(j, S)^{2}-\sum_{j=1}^{t} \omega(j, t)^{2}\right]-\sum_{j=1}^{t}[\omega(j, S)-\omega(j, t)] \eta_{j}-\log \left[\frac{B^{*}(0, t)}{B^{*}(0, S)}\right]\right]}{B^{*}(t, S)} \times \\
& E_{t}^{\mathbb{Q}}\left[\exp \left(-\sum_{j=t+2}^{S} \omega(j, S) \eta_{j}-\omega(t+1, S) \eta_{t+1}+u\left[\nu^{*}+\varphi^{*^{\prime}} X_{t}+\sigma^{*} \eta_{t+1}\right]\right)\right] \\
= & k_{t, S} E_{t}^{\mathbb{Q}}\left[\exp \left(-\sum_{j=t+2}^{S} \omega(j, S) \eta_{j}\right)\right] \times \\
= & k_{t, S}^{\prime} \exp \left[u \left[\nu_{t}^{\mathbb{Q}}\left[\exp \left(u\left(\nu^{*}+\varphi^{*^{\prime}} X_{t}\right)+\left(u \sigma^{*}-\omega(t+1, S)\right) \eta_{t+1}\right)\right]\right.\right.
\end{aligned}
$$

now, using (29) we have that

$$
\begin{aligned}
k_{t, S} & =\frac{\exp \left[-\frac{1}{2}\left[\sum_{j=1}^{S} \omega(j, S)^{2}-\sum_{j=1}^{t} \omega(j, t)^{2}\right]-\sum_{j=1}^{t}[\omega(j, S)-\omega(j, t)] \eta_{j}-\log \left[\frac{B^{*}(0, t)}{B^{*}(0, S)}\right]\right]}{B^{*}(t, S)} \\
& =\exp \left[-\frac{1}{2} \sum_{j=t+1}^{S} \omega(j, S)^{2}\right]
\end{aligned}
$$

and that 


$$
k_{t, S}^{\prime}=k_{t, S} \exp \left[\frac{1}{2} \sum_{j=t+1}^{S} \omega(j, S)^{2}\right]=1 .
$$

Consequently, Proposition 13 is proved.

\section{Appendix 9 : Proof of Proposition 14}

$$
\begin{aligned}
\rho(t+1, T) & =\log \left[B^{*}(t+1, T)\right]-\log \left[B^{*}(t, T)\right] \\
& =c_{T-t-1}^{\prime} X_{t+1}+d_{T-t-1}-c_{T-t}^{\prime} X_{t}-d_{T-t} \\
& =c_{T-t-1}^{\prime}\left[X_{t+1}-\Phi^{*} X_{t}-\tilde{\nu}^{*}\right]+r_{t+1}-\frac{1}{2} c_{1, T-t-1}^{2} \sigma^{* 2} \\
& =c_{T-t-1}^{\prime}\left[\sigma^{*}\left(\tilde{\xi}_{t+1}-\omega(t+1, S) e_{1}\right)\right]+r_{t+1}-\frac{1}{2} c_{1, T-t-1}^{2} \sigma^{* 2} \\
& =r_{t+1}+\omega(t+1, T) \omega(t+1, S) \\
& \quad-\frac{1}{2} \omega(t+1, T)^{2}-\omega(t+1, T) \xi_{t+1} .
\end{aligned}
$$

\section{Appendix 10 : Proof of Proposition 15}

Let us consider the $S$-forward probability $\mathbb{Q}_{S}$ determined by the numeraire $N_{t}=\frac{B^{*}(t, S)}{B^{*}(0, S)}$, with $t \leq T \leq S$. We have seen in Proposition 14 that :

$$
\rho(t+1, T)=r_{t+1}-\frac{1}{2} \omega(t+1, T)^{2}+\omega(t+1, T) \omega(t+1, S)-\omega(t+1, T) \xi_{t+1},
$$

and, consequently, the log-price zero-coupon bond process is given by :

$$
\begin{gathered}
\log \left[B^{*}(t, T)\right]=-\sum_{j=1}^{t} \omega(j, T) \xi_{j}+\sum_{j=1}^{t} \omega(j, T) \omega(j, S)+\sum_{j=1}^{t} r_{j} \\
-\frac{1}{2} \sum_{j=1}^{t} \omega(j, T)^{2}+\log \left[B^{*}(0, T)\right] .
\end{gathered}
$$

If we put $T=t$ in (A.16), we find the following relation for the sum of the short-rates :

$$
\sum_{j=1}^{t} r_{j}=\sum_{j=1}^{t} \omega(j, t) \xi_{j}-\sum_{j=1}^{t} \omega(j, t) \omega(j, S)+\frac{1}{2} \sum_{j=1}^{t} \omega(j, t)^{2}-\log \left[B^{*}(0, t)\right],
$$

that we can substitute in (A.16) to find :

$$
\begin{aligned}
& \log \left[B^{*}(t, T)\right]=\log \left[\frac{B^{*}(0, T)}{B^{*}(0, t)}\right]+\sum_{j=1}^{t}[\omega(j, t)-\omega(j, T)] \xi_{j} \\
&-\sum_{j=1}^{t} \omega(j, S)[\omega(j, t)-\omega(j, T)] \\
&+\frac{1}{2} \sum_{j=1}^{t}\left[\omega(j, t)^{2}-\omega(j, T)^{2}\right] .
\end{aligned}
$$




\section{Appendix 11 : Proof of Proposition 18}

We write relation (11) in the following way:

$$
R(t, h)=\mathbf{C}_{h}(L) x_{t}+\delta_{h},
$$

where $\mathbf{C}_{h}(L)=-\left(c_{1, h}+c_{2, h} L+\ldots+c_{p, h} L^{p-1}\right) / h$ is the $(p-1)^{t h}$ degree polynomial in the backward shift operator $L$, and where $\delta_{h}=-\left(d_{h} / h\right)$. Now, if we apply on the right-hand and left-hand side of this relation the operator $\Psi(L)=\left(1-\varphi_{1} L-\ldots \varphi_{p} L^{p}\right)$ operating on $t$, we can write :

$$
\begin{aligned}
\Psi(L) R(t, h) & =\mathbf{C}_{h}(L) \Psi(L) x_{t}+\Psi(1) \delta_{h} \\
& =\mathbf{C}_{h}(L)\left[\nu+\sigma \varepsilon_{t}\right]+\Psi(1) \delta_{h}=\sigma \mathbf{C}_{h}(L) \varepsilon_{t}+\mathbf{C}_{h}(1) \nu+\Psi(1) \delta_{h}
\end{aligned}
$$

\section{Appendix 12 : Proof of Proposition 22}

Assuming that (74) is true for $h-1$, we get:

$$
\begin{aligned}
& B(t, h)=\exp \left(c_{h}^{\prime} X_{t}+d_{h}\right) \\
&=E_{t}\left[M_{t, t+1} \cdots M_{t+H-1, t+H}\right] \\
&=E_{t}\left[M_{t, t+1} B(t+1, h-1)\right] \\
&=\exp \left[-\beta-\alpha^{\prime} X_{t}-\frac{1}{2} \Gamma_{t}^{\prime} \Gamma_{t}+d_{h-1}\right] \times E_{t}\left[\exp \left(\Gamma_{t}^{\prime} \varepsilon_{t+1}+c_{h-1}^{\prime} X_{t+1}\right)\right] \\
&=\exp \left[-\beta-\alpha^{\prime} X_{t}-\frac{1}{2} \Gamma_{t}^{\prime} \Gamma_{t}+d_{h-1}+c_{h-1}^{\prime} \Phi X_{t}+c_{1, h-1}^{\prime} \nu\right] \\
& \quad\left.\times E_{t}\left[\exp \left(\Gamma_{t}+\sigma^{\prime} c_{1, h-1}\right)^{\prime} \varepsilon_{t+1}\right)\right] \\
&=\exp \left[\left(-\alpha+\Phi^{\prime} c_{h-1}+(\sigma \gamma)^{\prime} c_{1, h-1}\right)^{\prime} X_{t}\right. \\
&\left.\quad+\left(-\beta+c_{1, h-1}^{\prime}(\nu+\sigma \gamma)+\frac{1}{2} c_{1, h-1}^{\prime} \sigma \sigma^{\prime} c_{1, h-1}+d_{h-1}\right)\right]
\end{aligned}
$$

and by identifying the coefficients we find the recursive relation presented in Proposition 22 . 


\section{R E F E R E N C E S}

Ang, A., Piazzesi, M., and M. Wei, (2006) : "What does the Yield Curve tell us about GDP Growth", Journal of Econometrics, 131, 359-403.

Berardi, A., (2005) : "Term Structure, Inflation and Real Activity", Working Paper, University of Verona.

Berardi, A., and W. Torous, (2005) : "Term Structure Forecasts of Long Term Consumption Growth", Journal of Financial and Quantitative Analysis, 40(2), 241-258.

Black, F., and P. Karasinsky, (1991) : "Bond and Option Pricing when Short Rates are Lognormal", Financial Analysts Journal, 47, 52-59.

Brandt, M. W., and D. A. Chapman, (2002) : "Comparing Multifactor Models of the Term Structure", Working Paper.

Brigo, D., and F. Mercurio, (2006) : "Interest Rate Models - Theory and Practice. With Smile, Inflation and Credit", Springer Finance.

Chen, L. (1996) : "Stochastic Mean and Stochastic Volatility - A Three-Factor Model of the Term Structure of Interest Rates and Its Application to the Pricing of Interest Rate Derivatives", Blackwell Publishers, Oxford, U.K..

Cheridito, R., Filipovic, D., and R. Kimmel, (2005) : "Market Prices of Risk in Affine Models: Theory and Evidence", forthcoming Journal of Financial Economics.

Cochrane, J. (2005) : "Asset Pricing", Princeton University Press.

Cox, J.C., Ingersoll, J.E. and S. A. Ross (1985) : "A theory of the term structure of interest rates", Econometrica, 53(2), pp. 385-407.

Dai Q., and K. Singleton (2000) : "Specification Analysis of Affine Term Structure Models", Journal of Finance, 55, 1943-1978.

Duarte, J., (2004) : "Evaluating An Alternative Risk Preference in Affine Term Structure Models", Review of Financial Studies, 17, 379-404.

Duffee, G. R., (2002) : "Term Premia and Interest Rate Forecasts in Affine Models", Journal of Finance, 57, 405-443.

Duffie, D., and R., Kan (1996) : "A yield-factor model of interest rates", Mathematical Finance, $6,379-406$.

Dybvig, P. H., (1988) : "Bond and Bond Option Pricing Based on the Current Term Structure", Working Paper, Washington University.

Gourieroux, C., and A. Monfort, (2006) : "Econometric Specifications of Stochastic Discount Factor Models", forthcoming Journal of Econometrics. 
Gourieroux, C., Monfort, A., and V. Polimenis, (2003) : "Discrete Time Affine Term Structure Models", Crest DP.

Gourieroux, C., Monfort, A., and V. Polimenis, (2006) : "Affine Model for Credit Risk Analysis", Journal of Financial Econometrics, 4, 3, 494-530.

Gourieroux, C., and R. Sufana, (2003) : "Wishart Quadratic Term Structure Models", Working Paper.

Heath, D., Jarrow, R. and A. Morton (1992) : "Bond Pricing and the Term Structure of Interest Rates: A New Methodology for Contingent Claims Valuation", Econometrica, 60, 77-105.

Ho, T., and S. Lee (1986) : "Term Structure Movements and Pricing Interest Rate Contingent Claims", Journal of Finance, 41, 1011-1028.

Hull, J., and A. White, (1990) : "Pricing Interest Rate Derivative Securities", The Review of Financial Studies, 3, 573-592.

Hull, J., and A. White, (1994) : "Branching Out", Risk, 7, 34-37.

Jamshidian, F., (1995) : "A Simple Class of Square-Root Interest Rate Models", Applied Mathematical Finance, 2, 61-72.

Monfort, A., and F. Pegoraro (2006) : "Switching VARMA Term Structure Models", forthcoming Journal of Financial Econometrics. Verlag.

Musiela, M., and M. Rutkowski (2005) : "Martingale Methods in Financial Modelling", Springer

Pearson, N. D., and T.-S. Sun, (1994) : "Exploiting the Conditional Density in Estimating the Term Structure : An Aplication to the Cox, Ingersoll and Ross Model", Journal of Finance, 49, 1279-1304.

Pegoraro, F., (2006) : "Discrete Time Factor Models for Asset Pricing", PhD Thesis, Université Paris-Dauphine (France).

Pennacchi, G., (1991) : "Identifying the dynamics of real interest rates and inflation: evidence using survey data", Review of Financial Studies, 4, 53-86.

Polimenis, V. (2001) : "Essays in Discrete Time Asset Pricing", Ph. D. Thesis, Wharton School, University of Pennsylvania.

Vasicek, O. (1977) : "An Equilibrium Characterization of the Term Structure", Journal of Financial Economics, 5, 177-188. 
Yield to Maturity
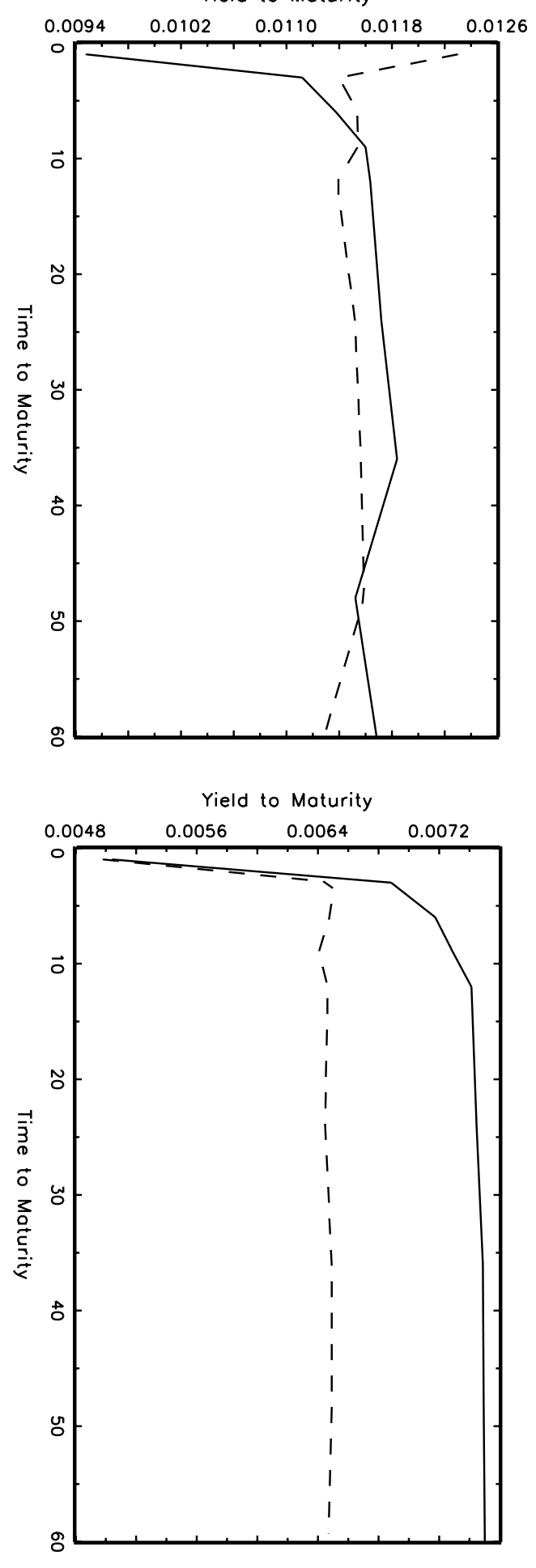

Yield to Maturity
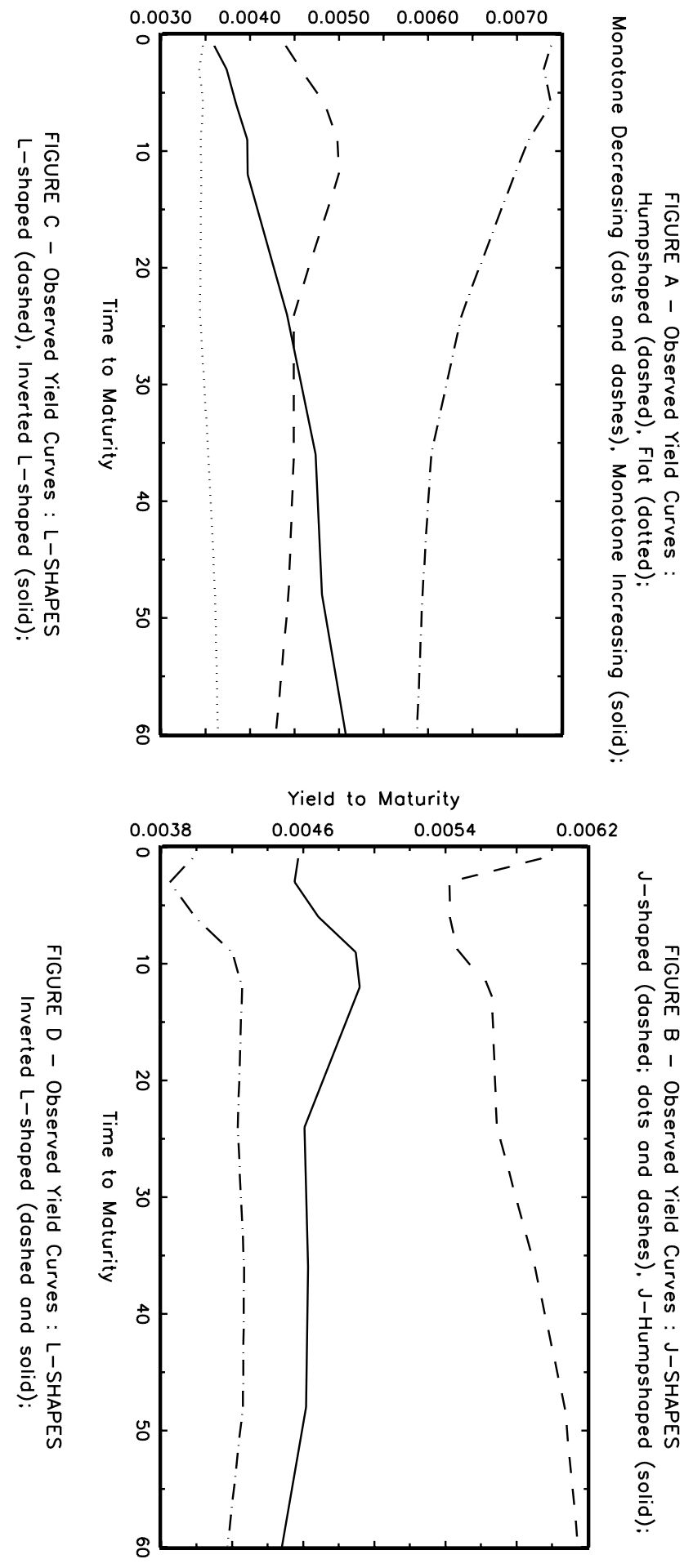

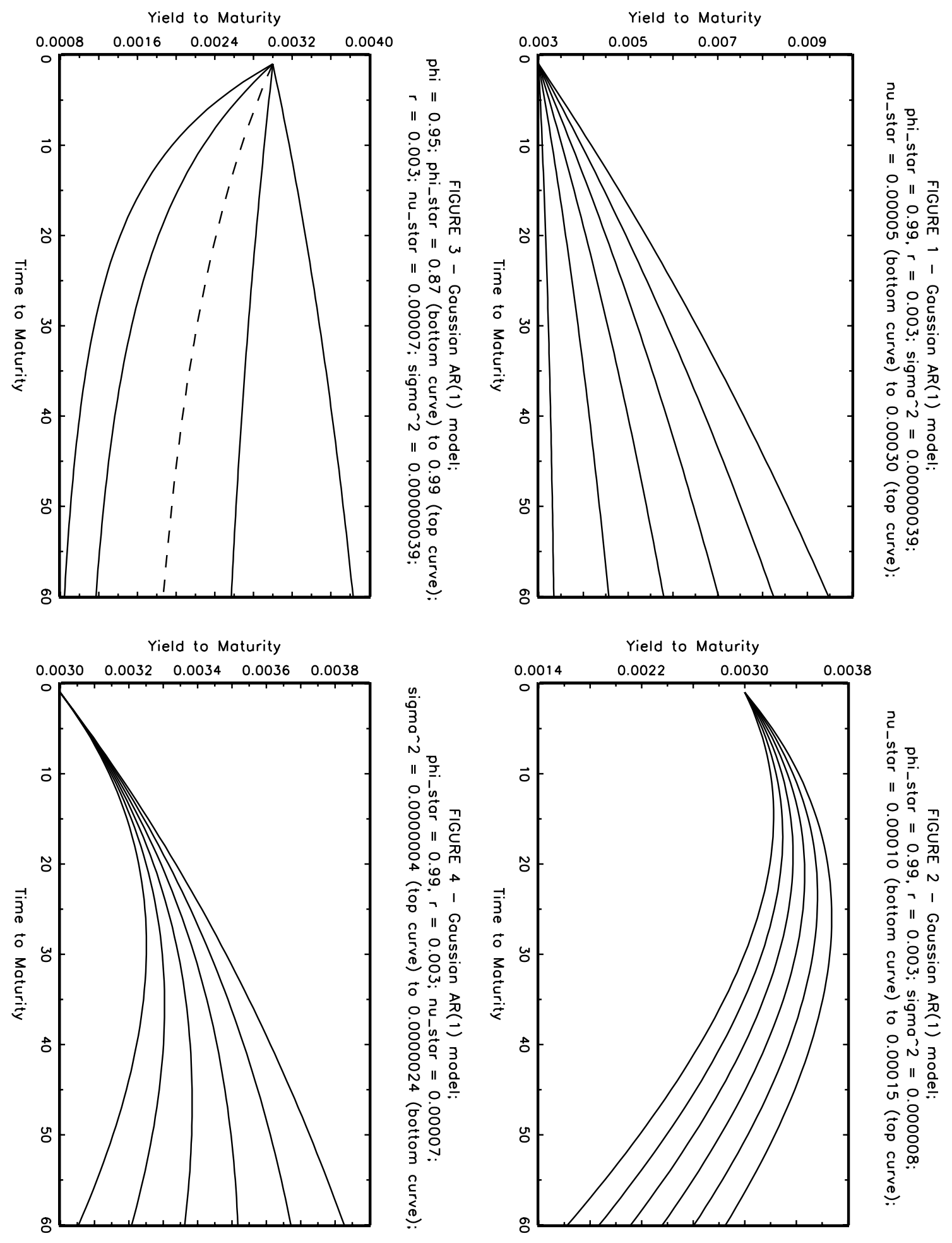

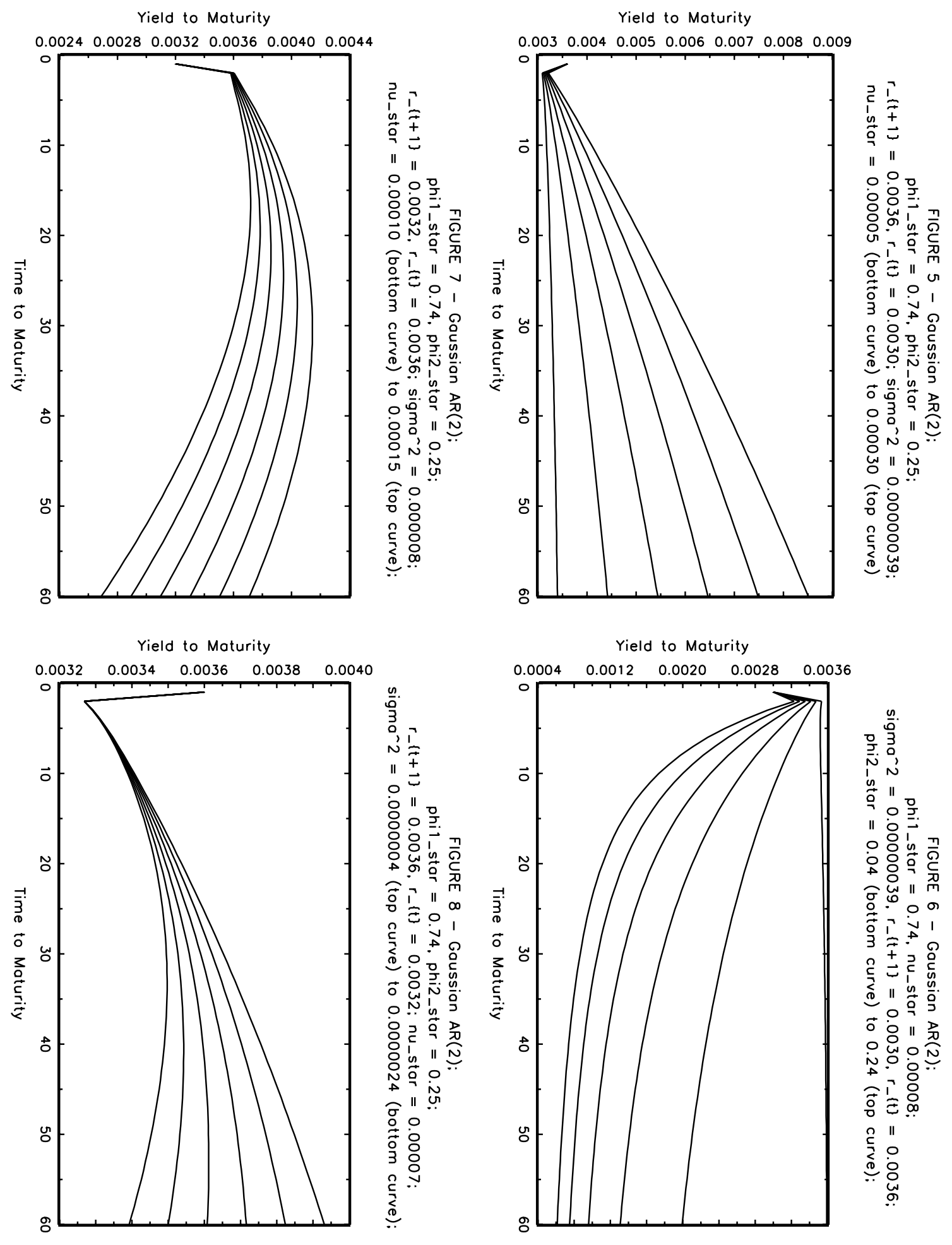

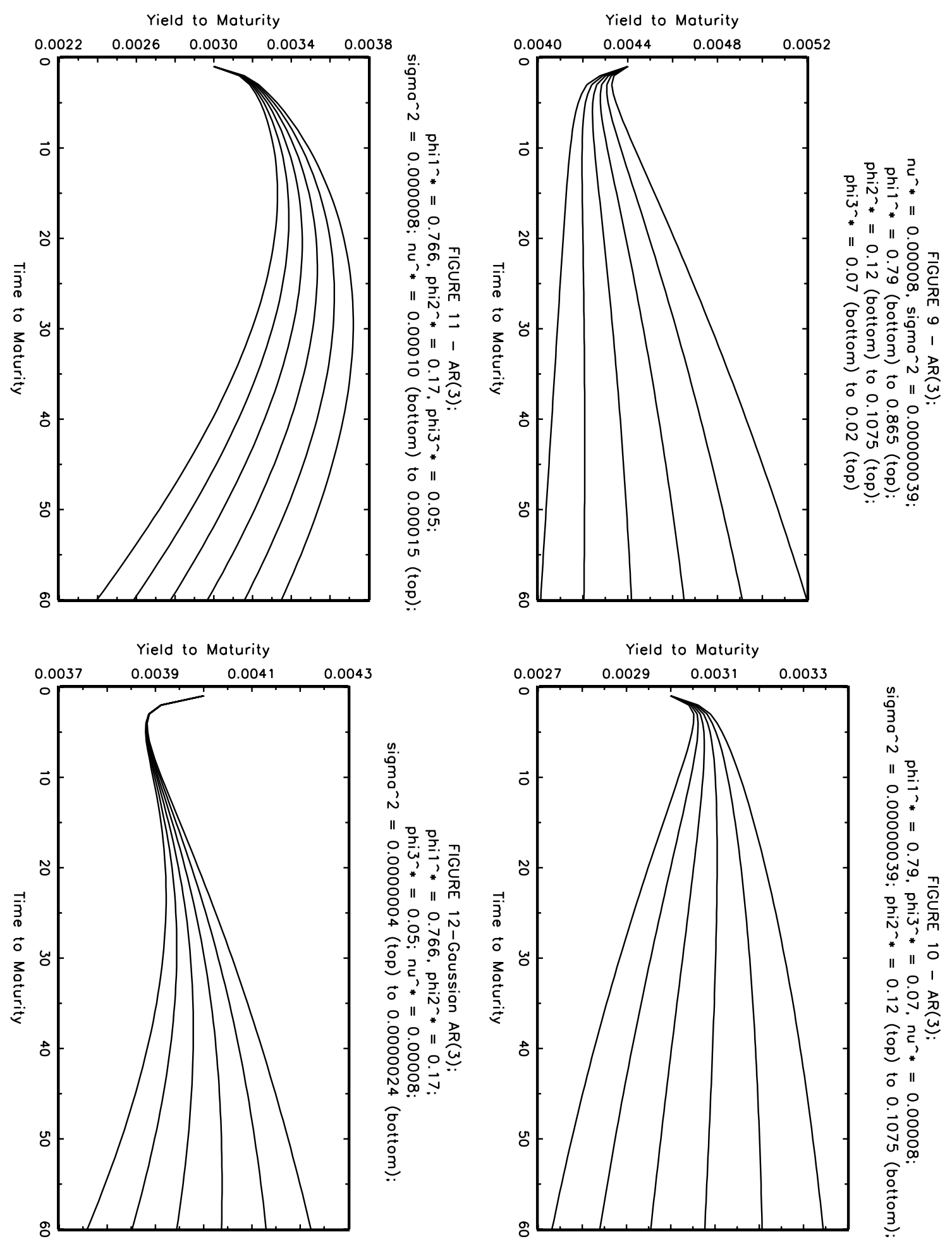

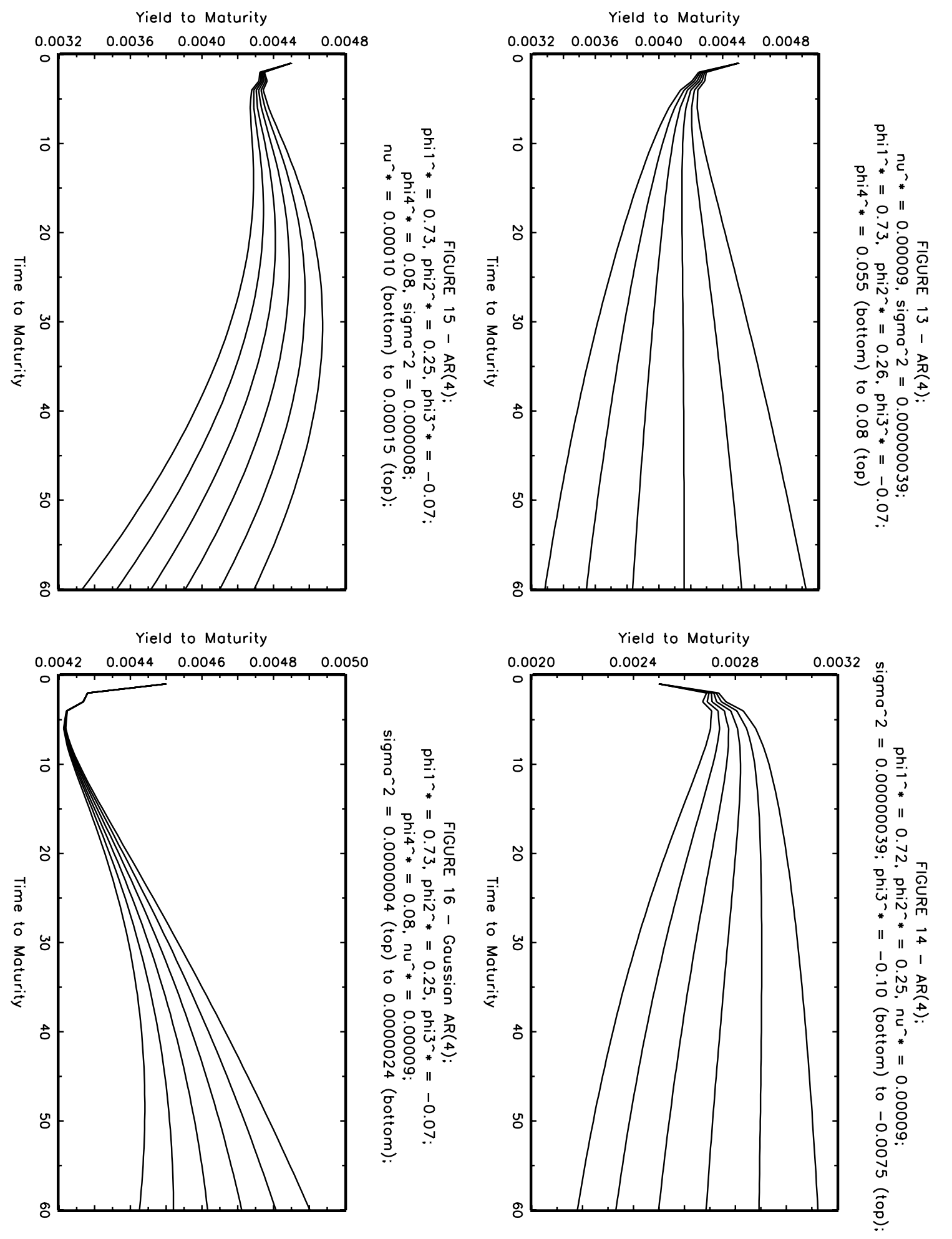